\title{
Upscaling Organic Electronic Devices
}

Abdellah Malti 
Linköping studies in science and technology. Dissertation No. 1711 2015 (D) All rights reversed.

Printed by LiU-Tryck, Linköping, Sweden, 2015

ISBN 978-91-7685-929-2

ISSN 0345-7524 
"Dissent is the native activity of the scientist [...].

To me, being an intellectual doesn't mean knowing about intellectual issues, it means taking pleasure in them."

- Jacob Bronowski (1908-1974) 



\section{Dedication}

To Humanity.

The known-universe's best hope to understand Itself, Relentless challenger of entropy, Champion of Truth and Freedom;

Lest We fall prey to the perilous comforts of relativism. 



\section{Abstract}

Conventional electronics based on silicon, germanium, or compounds of gallium require prohibitively expensive investments. A state-of-the-art microprocessor fabrication facility can cost up to $\$ 15$ billion while using environmentally hazardous processes. In that context, the discovery of solution-processable conducting (and semiconducting) polymers stirred up expectations of ubiquitous electronics because it enables the mass-production of devices using well established high-volume printing techniques.

In essence, this thesis attempts to study the characteristics and applications of thin conducting polymer films $(<200 \mathrm{~nm})$, and scale them up to thick-films $(>100 \mu \mathrm{m})$. First, thin-films of organic materials were combined with an electric double layer capacitor to decrease the operating voltage of organic field effect transistors. In addition, ionic current-rectifying diodes membranes were integrated inside electrochromic displays to increase the device's bistability and obviate the need for an expensive addressing backplane.

This work also shows that it is possible to forgo the substrate and produce a self-standing electrochromic device by compositing the same water-processable material with nanofibrillated cellulose (plus a whitening pigment and high-boiling point solvents). In addition, we investigated the viability of these (semi)conducting polymer nanopaper composites in a variety of applications. This material exhibited an excellent combined electronic-ionic conductivity. Moreover, the conductivities in this easy-to-process composite remained constant within a wide range of thicknesses. Initially, this (semi)conducting nanopaper composite was used to produce electrochemical transistors with a giant transconductance $(>1 \mathrm{~S})$. Subsequently, it was used as electrodes to construct a supercapacitor whose capacitance exceeds $1 \mathrm{~F}$. 



\section{Contribution to scholarly articles}

- Ultra-low voltage air-stable polyelectrolyte gated ntype organic thin film transistors

A. Malti, E. O. Gabrielsson, M. Berggren, X. Crispin

Appl. Phys. Lett. 99, 063305 (2011)

Contribution: Most of the experimental work and most of the writing/editing of the manuscript.

- Low-voltage ambipolar polyelectrolyte-gated organic thin film transistors

A. Malti, M. Berggren and X. Crispin

Appl. Phys. Lett. 100, 183302 (2012)

Contribution: All experimental work and most of the writing/editing of the manuscript.

- An Electrochromic Bipolar Membrane Diode A. Malti, E. O. Gabrielsson, X. Crispin, M. Berggren Adv. Mater. 27, 3909-3914 (2015)

Contribution: Most of the experimental work and most of the writing/editing of the manuscript.

- A substrate-free electrochromic device

A. Malti, R. Brooke, X. Liu, D. Zhao, P. Andersson Ersman, M. Fahlman, M. Berggren, X. Crispin

Submitted (Aug. 2015) 
Contribution: Most of the experimental work and most of the writing/editing of the manuscript.

- Enabling organic power electronics with a cellulose nano-scaffold

A. Malti, J. Edberg, H. Granberg, Z. Khan, J. Andreasen, X. Liu, D. Zhao, H. Zhang, Y. Yao, J. Brill, I. Engquist, M. Fahlman, L. Wåberg, X. Crispin, M. Berggren

Submitted (Sep. 2015)

Contribution: Some of the experimental work and some of the writing/editing of the manuscript. 


\section{Scholarly articles \\ beyond the scope of this thesis}

- Optimization of the thermoelectric figure of merit in the conducting polymer poly(3,4-ethylenedioxythiophene) O. Bubnova, Z. Khan, A. Malti, S. Braun, M. Fahlman, M. Berggren, X. Crispin

Nature Mater. 10, 429-433 (2011)

Contribution: Preliminary experimental work and minor involvement in the writing/editing of the manuscript. 



\section{Contents}

\section{Background}

1 Introduction 1

2 Materials $\mathbf{5}$

2.1 Conjugated Polymers . . . . . . . . . . . . 5

2.1.1 Atomic Orbitals . . . . . . . . . . 5

2.1.2 Molecular Orbitals . . . . . . . . . . 6

2.1.3 Polymers ............... 8

2.1.4 Conjugated Polymers . . . . . . . . 9

2.1.5 Charge Carriers in Conjugated Polymers . . . 11

2.1.6 Doping of conjugated polymers . . . . . . . . 14

2.1.7 Electrochromism in conjugated polymers . . 15

2.2 Polyelectrolytes . . . . . . . . . . . . . . . . . 17

2.2.1 Electrolytes . . . . . . . . . . . . . . . 17

2.2.2 Polyelectrolytes . . . . . . . . . . 17

2.2.3 Charge transport in polyelectrolytes . . . . . 18

2.2.4 Ionic selectivity . . . . . . . . . . . . . 19

2.3 Cellulose . . . . . . . . . . . . . . . . . . . . . . . 20

2.3.1 Manufacturing ............ . 21

2.3.2 Cellulose nanofibers .......... . 21

3 Devices 23

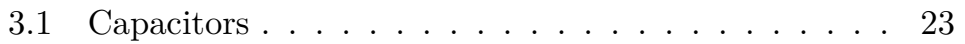

3.1.1 Solid dielectric capacitor . . . . . . . . . 23

3.1.2 Electrostatic double layer capacitor . . . . . 25

3.1.3 Electrochemical pseudocapacitor . . . . . . . 27

3.2 Organic electrochromic display . . . . . . . . . 28

3.3 Bipolar membrane diode . . . . . . . . . . . . 28

3.3.1 Diode .................. . . 28

3.3.2 Bipolar membrane ........... 30 


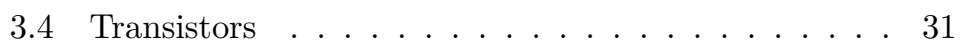

3.4.1 Overview ................. 31

3.4.2 Bipolar junction transistor . . . . . . . 33

3.4.3 Field-effect transistor . . . . . . . . . . 33

3.4.4 Organic electrochemical transistor . . . . . . 40

4 Methods 43

4.1 Manufacturing techniques . . . . . . . . . . . 43

4.1.1 Spin-coating . . . . . . . . . . . . 43

4.1 .2 Photolithograhy ............. . . 44

4.1 .3 Solution casting . . . . . . . . . . . . 45

4.2 Electrochemistry . . . . . . . . . . . . . . 45

4.2.1 Cyclic voltammetry . . . . . . . . . . 47

4.2.2 Impedance spectroscopy . . . . . . . . . . 48

5 Conclusions $\quad 51$

II Scholarly articles 


\section{Part I}

\section{Background}





\section{Chapter 1}

\section{Introduction}

Synthetic polymers (commonly referred to as plastics) have been intricately associated with modernity and progress throughout the $20^{\text {th }}$ century. Spurred by the second world war's boom (no pun intended) in chemical technology and the commercial successes of early thermoplastic/thermoset resins - such as celluloid, bakelite, polyvinyl chloride (PVC), polystyrene (PS) and aliphatic polyamides (Nylon) - plastics exploded in variety and popularity in the 1960s. Synthetic polymers are such a part and parcel of modern society that it is hard to imagine daily life without them. These materials' ubiquity and irreplaceability will no doubt be magnified once 3D printing (i.e. additive manufacturing) becomes a mainstream technology.

Among the many advantages of synthetic polymers, one may cite affordability, recyclability, customizability and versatility.

Polymer products are affordable because the raw materials required for their synthesis (cellulose, coal, natural gas, salt and crude oil waste) are abundant the world over. From a chemistry perspective, the polymerization processes are simple, safe and wellunderstood. Economies of scale help drive the price of polymers ever lower since continuous-flow manufacturing is quasi-optimal for mass production. Furthermore, plastic products may be recycled by melting and sorting them into pellets of different densities to make new (most often, entirely different) products. This makes plastics very competitive when stacked against materials that cannot be reprocessed as easily.

Polymers are also highly customizable and versatile in the sense that it is possible to tweak their mechanical, optical, thermal, sol- 
uble, and electrical properties. This aspect of polymers happens to be a boon for mass-customization.[1] It enables the manufacturing of objects with customized shape, density, hardness, color, resistance to heat and solubility.

Thanks to the pioneering work of Heeger, Shirikawa and McDiarmid on (semi)conducting polymers in the late 1970s, it is possible to obtain electronic conductivity in certain polymer families (namely, conjugated polymers in which there's an alternation of single and double bonds).[2] The trio's research spurred considerable academic and industrial interest in the field of "organic ${ }^{1}$ electronics". Initially, this discovery created a research area at the intersection of chemistry and condensed matter physics. Ultimately, Heeger, Shirikawa and McDiarmid were jointly awarded the Nobel prize in chemistry for the year 2000.[3] Nowadays, organic electronics has matured into an established research discipline within materials science. Because of their solubility/emulsifiability in common solvents - and therefore, compatibility with printing techniques - conjugated polymers are regarded as a highly promising route to produce low-cost ubiquitous electronic devices.[4] Organic electronics offers many advantages over conventional (i.e. silicon-based) electronics, namely biocompatibility, flexibility and biodegradability. [5] Note that the biocompatibility claims rely too often on little more that a fallacious conflation which exploits the polysemy of the word organic.

The energy required to produce electronic consumables is unintuitively large. The global production of electronic-grade silicon consumes dozens of petajoules. ${ }^{2}$ The transformation of these monocrystalline ingots into modern electronic devices is an astoundingly energy-hungry process and an often overlooked argument in favor of embracing the organic electronics technology.[6] It is even claimed that more energy goes into building the average laptop/smartphone than an 80s/90s car.[5]

Prior to the discovery of electronically conducting polymers, solid electrolytes were already attracting attention because of their potential in energy storage applications. In the early 1970s, Fenton, Parker and Wright reported the first polymer electrolyte (a subset of solid electrolytes).[7] The first polymer electrolyte consisted of a semicrystalline structure polyethylene oxide (PEO) and alkali salt complexes. $[8,9,10]$ Polymer electrolytes are defined by

\footnotetext{
${ }^{1}$ in the chemical sense of the word, organic refers to a compound which contains a significant amount of carbon

${ }^{2} 1$ petajoule $=10^{15} \mathrm{~J}$
} 
the IUPAC as polymers "composed of macromolecules in which a substantial portion of the constitutional units contains ionic or ionizable groups, or both". Essentially, any supramolecular system doped with ions to improve the electrical conductivity may be regarded as a polymer electrolyte.[11] In polyelectrolytes, one of the ions is covalently bound to the polymer backbone while the counterion is mobile. As an application example, a polyelectrolyte may be utilized to create electric double layer capacitors (also known as supercapacitors, because they generate very large electric fields). The latter are made by simply sandwiching a polyelectrolyte between two conducting electrodes.

Efforts to scale up the production of electronic devices based on organic materials have so far focused on large-area applications. However, little has been done to explore the feasibility of scaling up organic materials (and devices) beyond thin-film technology. Even less investigative work delved into the properties and potential applications of thicker $(>1 \mu \mathrm{m})$ films of conducting polymers. In the latter part of this work, nanofibrillated cellulose (i.e. cellulose with nanometer-scale features) was used as a scaffold to produce electronically and ionically active substrates. Cellulose is a polysaccharide which gives plants their structural integrity. A series of devices are presented which exploit the electronic (semi)conduction, ionic conduction, ionic current rectification, ionic doping, electrochromism, flexibility and adhesiveness of polymers.

This thesis aims to provide the reader with the requisite background to understand, interpret and judge the research contained in the appended scholarly articles. All the while, it attempts to investigate the potential of upscaling organic materials and devices into the z-dimension $(>100 \mu \mathrm{m})$. The thesis starts with a brief introduction into the physics and chemistry of conjugated polymers, polyelectrolytes, and nanofibrillated cellulose. Thereafter, the reader is introduced to a series of electronic and ionic devices which exploit the properties of the materials introduced. The different experimental methods used to fabricate and characterize said devices are also touched upon. Finally, an educated guess about the potential impact of this work on future research and technologies is ventured. 


\section{Chapter 2}

\section{Materials}

\subsection{Conjugated Polymers}

\subsubsection{Atomic Orbitals}

As quantum theory matured post-1925, the popular planetarymodel analogy of electrons gravitating around the atomic nucleus (also known as the Rutherford-Bohr model) was displaced by a less-intuitive model wherein electrons occupy oddly-shaped "atmospheres" surrounding the nucleus.

An atom's nucleus and electron(s) can be described with the wavefunction $\psi(r, t)$. The probability of finding each elementary particle at location $r$ and time $t$ is represented by the density function $|\psi(r, t)|^{2}$. The electrons of an isolated atom may only occupy certain quantum states which correspond to discrete energy levels. These states are given by the allowed solutions of the Schrödinger equation called atomic orbitals. Only two atoms (at most) of opposing spin may occupy the same orbital simultaneously. Atomic orbitals are defined by shells (K, L, M, etc.) and orbital type (1, 2,3 , etc.).

Carbon's simplest atomic orbitals (1s, 2s and $2 p)$ also happen to be the most relevant to the field of organic electronics. Figure 2.1 shows the shape of an $s$ and a $p_{z}$ orbital. Carbon is midway along the first eight-element row in the periodic table with a moderate tendency to attract electrons. 


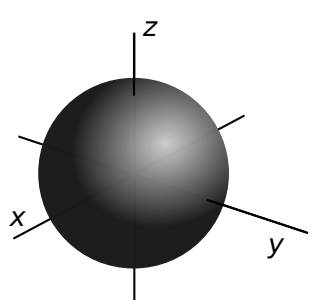

(a) s orbital.

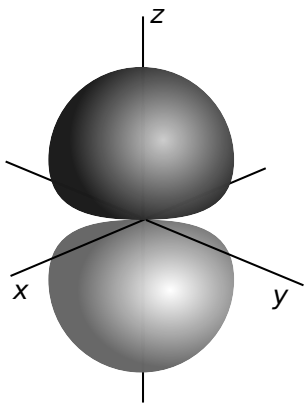

(b) $p_{z}$ orbital.

Figure 2.1: Shape of common molecular orbitals.

\subsubsection{Molecular Orbitals}

The outermost shell of an atom is called the valence shell. When two atoms are in the vicinity of one another, their valence electrons start to interact as their valence orbitals overlap resulting in molecular orbitals. Various types of molecular orbitals are constructed depending on which atomic orbitals formed them. The interaction between atomic orbitals may be either constructive or destructive (see figure $2.2^{1}$ ). A constructive interaction increases the electronic density around the nuclei leading to the formation of a bonding orbital. A destructive interaction, however, decreases the aforementioned electronic density and is known as an antibonding orbital. A bonding orbital has lower energy than the combined original atomic orbitals and, consequently, has a stabilizing effect on the molecule. The opposite effect holds true for an antibonding orbital. Electrons are added to molecular orbitals, one at a time, starting with the lowest energy molecular orbital. A stable bond between atoms is formed when the energy of the system is lower than that of the individual atoms.

When the number of bonding electron pairs exceeds the number of links between atoms (e.g. alkenes - introduced in the next section), the extra electrons occupy orbitals which are higher in energy than the orbitals of molecules where the number of bonding electron pairs is equal to the number of links between atoms. The former atoms are said to form double bonds. The orbital occupied by electrons with highest energy is called the Highest Occupied

\footnotetext{
${ }^{1} \mathrm{E}$ stands for Energy
} 


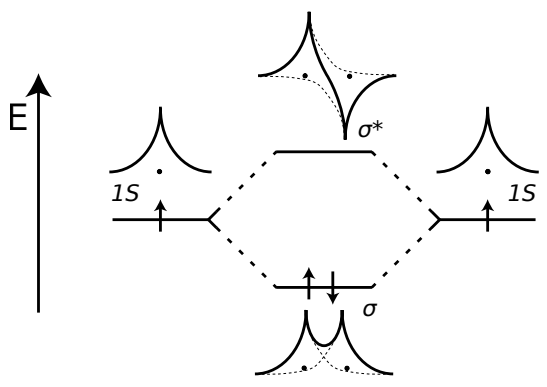

Figure 2.2: Energy diagram from the formation of bonding and antibonding molecular orbitals for a molecule composed of two 1s orbitals.

Molecular Orbital (HOMO). The unoccupied orbital with lowest energy (directly above the HOMO) is called the Lowest Unoccupied Molecular Orbital (LUMO).

Valence bond (VB) theory is a quantum mechanical approach to determine the structure of chemical bonds. The concept of hybridization was introduced to account for VB's failure to explain the difference between the predicted and measured bond angles of organic compounds. According to VB theory, carbon should form two covalent bonds making a $\mathrm{CH}_{2}$ molecule possible. The latter molecule has, however, proven elusive to (experimental) detection outside of a reaction. $\mathrm{CH}_{4}$, on the other hand, is found all over the universe. ${ }^{2}$ To explain the discrepancy between VB theory and experimental observations, the concept of hybridization was introduced.

The four $\sigma$ bonds linking the carbon to the hydrogen atoms in a $\mathrm{CH}_{4}$ molecule result from the hybridization (i.e. mixing) between the valence shell $s$ and $p$ wavefunctions. The energy levels of hybrid orbitals are identical. Furthermore, the hybridization of orbitals is energetically favorable. In addition, the frontal lobes of hybridized orbitals overlap better than their unhybridized counterparts, resulting in stronger bonds and more stable compounds. The carbon in a tetrahedrally coordinated carbon (such as in a $\mathrm{CH}_{4}$ molecule) is said to be $s p^{3}$ hybridized. Figure 2.3(a) illustrates the tetrahedral shape of said hybridized orbitals. In organic molecules that form double bonds $\left(\mathrm{C}_{2} \mathrm{H}_{4}\right.$ for example), the carbon atom orbitals are $s p^{2}$ hybridized. Figure 2.3(b) shows that the $s p^{2}$ carbon atoms

\footnotetext{
${ }^{2} \mathrm{CH}_{4}$, A.K.A. methane, is the main component of natural gas
} 


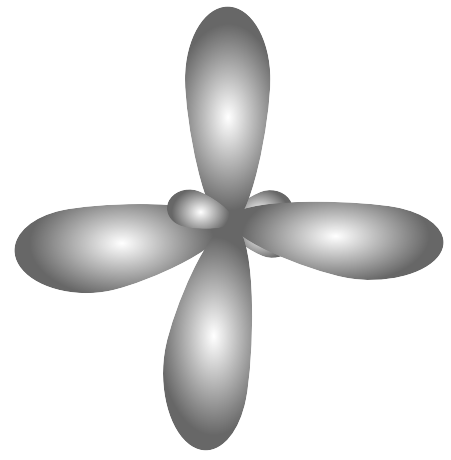

(a) Illustration of $\mathrm{sp}^{3}$ hybridized orbital.

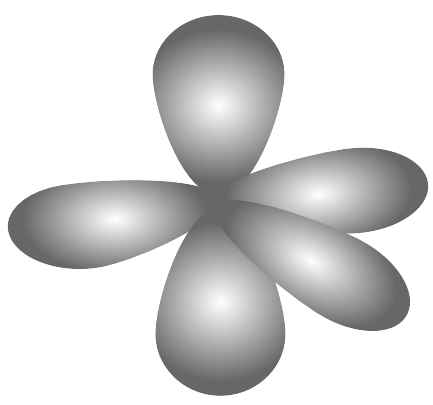

(b) Illustration of $\mathrm{sp}^{2}$ hybridized orbital.

Figure 2.3: Hybridized orbitals.

may only form three $\sigma$ bonds. A $\pi$ bond is formed when the $p$ orbitals - shown vertically in figure $2.3(\mathrm{~b})$ - combine with the $p$ orbitals from an adjacent carbon atom.

\subsubsection{Polymers}

A polymer is a substance composed of many repeating units (called monomers). ${ }^{3}$ DNA, cellulose and proteins are all examples of naturally occurring polymers. Owing to their virtually limitless number of designs, synthetic polymers have become synonymous with modernity and an integral part of the mass production process.

The simplest polymers from a chemical standpoint are hydrocarbons which are organic compounds made exclusively of carbon and hydrogen atoms. When atoms are in proximity to one another, their respective atomic orbitals combine to form molecular orbitals. As the size of a molecule grows, so does the number of molecular orbitals. The energy levels of such molecules become so densely packed that they may be viewed as a continuum of allowed energies called a band. A band gap describes the ranges of energy which occur outside the previously described continuum of allowed energies.

Carbon is a tetravalent atom with an incomplete outer shell, and may bond with a maximum of 4 hydrogen atoms. Table 2.1

\footnotetext{
${ }^{3}$ The suffix -mer is derived from meros, which is Greek for part. As polyglots often monotonously point out.
} 


\begin{tabular}{c|l|l|l} 
Number of carbons & Alkane & Alkene & Alkyne \\
\hline 1 & Methane & - & - \\
2 & Ethane & Ethylene & Acetylene \\
3 & Propane & Propylene & methylacetylene \\
4 & Butane & Butylene & Butyne
\end{tabular}

Table 2.1: Variations of simple hydrocarbons.

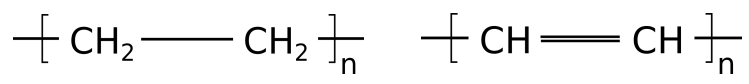

(a) polyethylene

(b) polyacetylene

Figure 2.4: Chemical structure of simple carbohydrate polymers

shows the alkane (single bond between the carbons), alkene (double bond) and alkyne (triple bond) versions of the simplest hydrocarbons.

\subsubsection{Conjugated Polymers}

Polyethylene is a long chain of ethylene monomers, where each carbon atom covalently binds to two atoms of carbons and two of hydrogens. The chemical structure of polyethylene is shown in 2.4(a). The material is best known for being extruded to make the common plastic shopping bag. ${ }^{4}$ Contrary to metals where electrons roam freely, the electrons in commonly-encountered polymers are tightly bound to the nuclei of the polymer chain. In the case of polyethylene, for example, the carbon valence electrons are localized in an $s p^{3}$ hybridized orbital. Polyethylene is an electrically insulating polymer as electrons in $\sigma$ bonds have very low mobility.

This is in contrast with the $s p^{2}$ carbon atoms in polyacetylene - depicted in figure 2.4(b) — which only form three $\sigma$ bonds. A conjugated system is one where there is an alternation between single and multiple bonds.[13] This feature generally results in a lower overall energy. It is worth mentioning that not all conjugated systems are polymers (e.g. graphene, carbon nanotubes, graphite, etc.).

A useful shorthand to depict polymers is shown in figure 2.5. Note that compared to figure 2.4, figure 2.5 doesn't show the indi-

\footnotetext{
${ }^{4}$ Celloplast, a Norrköping-based company, was issued a patent on the transformation of polyethylene into plastic bags through blown film extrusion.[12] The invalidation of that patent by Mobil coincided with Celloplast's demise.
} 


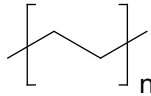

(a) polyethylene

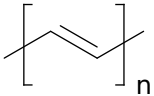

(b) polyacetylene

Figure 2.5: Shorthand representation of simple carbohydrate polymers

vidual atoms. Rather, every kink in the chain is assumed to contain a C atom.

As a consequence of their tetravalence, each carbon atom is left with a valence electron in a non-hybridized $p_{z}$ orbital perpendicular to the polymer backbone. The overlap of such (adjacent and parallel) p-orbitals along a polyacetylene chain forms a $\pi$ bond. The latter type of bonds are essentially delocalized electron clouds, and therefore are more diffuse than $\sigma$ bonds. In an alkene, the number of carbon atoms is proportional to the number of bonding and antibonding $\pi$ orbitals which, in turn, is proportional to the number of available energy levels. Figure 2.6 illustrates this splitting of energy levels of $\pi$ orbitals. As the number of carbon atoms doubles, the energy difference between $\pi$ orbitals decreases. For a sufficiently large number of atoms in a conjugated system, the energy difference between levels vanishes, turning the discrete energy levels into continuous bands. In essence, the presence of these bands is the basis of electronic conduction in conjugated polymers.

One-dimensional chains with equal bond length are unstable according to Peierls' theorem. In conjugated polymers, the alternation of bond lengths minimizes the energy, and therefore is the most likely configuration. This alternation stabilizes bonding orbitals while having the opposite effect on antibonding orbitals, which results in the energy splitting shown in figure 2.6. The top of the $\pi$ band may then be considered the HOMO of the conjugated system while the bottom of the $\pi^{*}$ corresponds to the LUMO. The difference between the HOMO and LUMO (i.e. $\mathrm{E}_{g}$ in figure 2.6) represents the band gap. The typical $\mathrm{E}_{g}$ value for a conjugated polymer is anywhere between 1.5 and $3 \mathrm{eV}$.[14] 


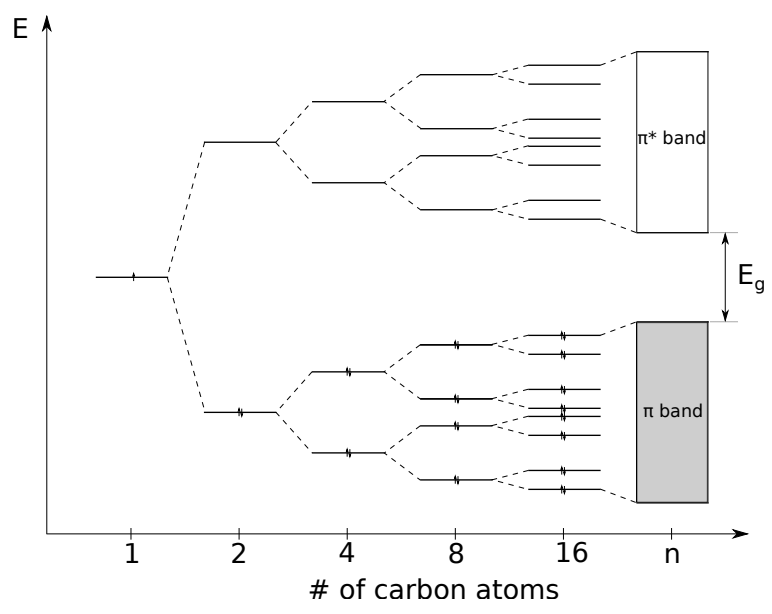

Figure 2.6: Energy splitting leading to band formation for sufficiently long conjugated polymers.

\subsubsection{Charge Carriers in Conjugated Polymers}

\subsubsection{Solitons}

An energy level is said to be degenerate if it corresponds to at least two distinct states of a quantum system. Thus, a conjugated polymer is said to have a degenerate ground state if switching the location of double and single bonds within it does not affect the energy of the system. In other words, when the alternation of $\mathrm{C}-\mathrm{C}$ and $\mathrm{C}=\mathrm{C}$ is reversed, the total energy curve of a degenerate polymer has two equal minima. Because their energies are identical, both polyacetylene configurations shown in figure 2.7 are equally likely to occur. When both configurations coexist on the same polyacetylene chain — as is the case in figure 2.8(a) — the transition region is distributed over several repeat units of the polymer chain. The boundary between the two phases is called a soliton. Solitons lead to localized electronic levels in the middle of the band gap, and are responsible for the charge transport in degenerate conjugated polymers. Figure 2.8(b) depicts a positive soliton. A neutral soliton, as its name implies, has no charge. Yet, despite consisting of an unpaired electron, it has spin $\frac{1}{2}$. A positive soliton, on the other hand, is charged but has no spin. 


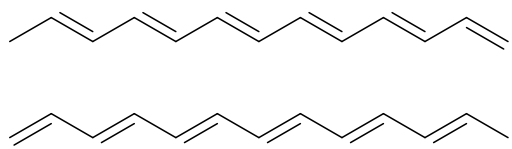

Figure 2.7: Polyacetylene configurations with an equal probability to occur due to the polymer's degenerate ground state.<smiles>C=CC=C(C)C=C=C=C=CC(C)=CC=C</smiles>

(a) Neutral soliton formation occurs at the transition region between two polyacetylene configurations.<smiles>C=CC=C(C)C=C=C=C=CC(C)=CC=C</smiles>

(b) Positive soliton.

Figure 2.8: Soliton formation in a polymer with degenerate ground state.

\subsubsection{Polarons}

Degenerate ground level conjugated polymers are, however, few and far between. Changing the alternation of single and double bonds in most conjugated polymers changes the total energy of the system, making one configuration more likely to occur than another. Polythiophenes are an example of a conjugated polymer with a non-degenerate ground state. The most stable form of polythiophene is the aromatic configuration shown in figure 2.9(a) where the double bonds reside inside aromatic rings. This ground state is disturbed when the single and double bonds are interchanged. This quinoid structure - shown in figure $2.9(\mathrm{~b})$ - is significantly less stable than the aromatic one.

Since polythiophenes have a non-degenerate ground level, there

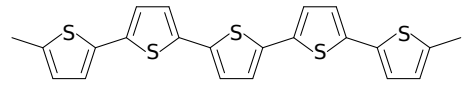

(a) Aromatic structure.

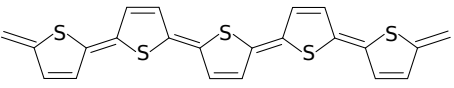

(b) Quinoid structure.

Figure 2.9: The different polyacetylene configurations. 


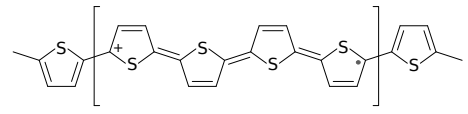

(a) Positive polaron.

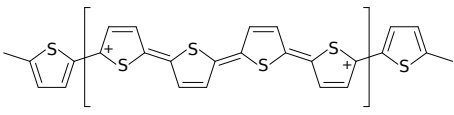

(b) Positive bipolaron.

Figure 2.10: Illustration of positively charged polarons and bipolarons.

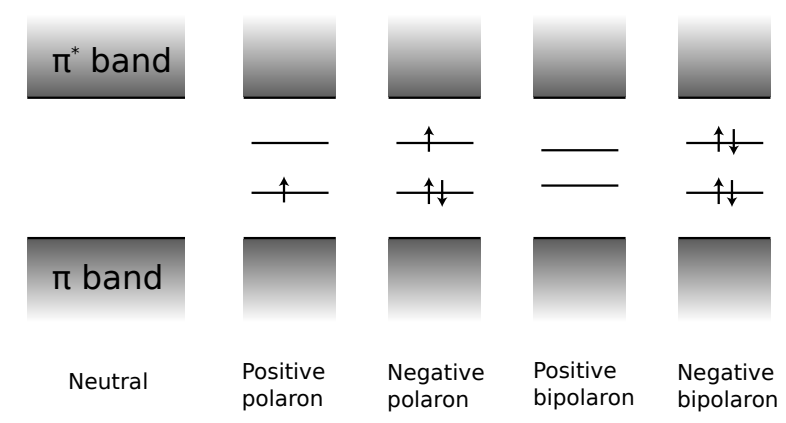

Figure 2.11: Band diagrams for polarons and bipolarons.

is a very low probability of finding the quinoid and aromatic structure spontaneously coexisting on the same polymer chain. Because of this, solitons are never encountered in polythiophenes. However, introducing a charge into the polymer chain (such as in the case of chemical oxidation) results in a local deformation of the surrounding bonds. This destabilization creates a charge carrier called a polaron. Figure 2.10(a) shows that, as for solitons, polarons are delocalized over a few repeat units of the polymer chain. When two polarons are close enough to each other, they may combine to form a bipolaron. Figure 2.10(b) depicts a bipolaron on a polythiophene chain. Polarons and bipolarons may be negative or positive depending on whether an electron was received or relinquished (respectively).

Polarons, bipolarons, as well as solitons are quasiparticles (sometimes, self-localized excitations).[15] That is, a simplified model to describe emergent phenomena in solid matter. ${ }^{5}$

\footnotetext{
${ }^{5}$ The need for these simplifications is eloquently crystallized by Max Born in the following statement: "It would indeed be remarkable if Nature fortified herself against further advances in knowledge behind the analytical difficulties of the many-body problem."
} 


\subsubsection{Interchain transport}

Solitons, polarons and bipolarons can move with ease along a polymer chain. To move farther than the length of a polymer chain, charge carriers typically resort to thermally activated interchain hopping. This transport process is the limiting factor for achieving high charge mobility in a conjugated polymer film. Increasing the overlap between the $\pi$ orbitals decreases the energy required for the charges to hop from one chain to another. Of course, the overall mobility of a polymer film is also affected by the material's purity and the density of its charge carriers.

A number of techniques (annealing, additives, etc.) are available to improve the crystallinity of a polymer film which, in turn, improves the intermolecular $\pi$ orbital overlap and charge mobility. The latter can reach up to $12 \mathrm{~cm}^{2} \mathrm{~V}^{-1} \mathrm{~s}^{-1}$ in highly ordered polymer semiconductors.[16] In these crystalline systems, the charge carriers are concentrated in localized states. These localized states are due to a high degree of randomness of impurities (or defects). The charge carrier's motion, on the other hand, happens in extended states which may be considered transport bands.

In amorphous systems, however, the charge mobility is more modest. A common way to model the interchain transport mechanism is variable-range hopping (VRH). According to the VRH model, the charge carriers require little energy to hop long distances, while they require considerably more energy to hop short ones.[17] Also, the mobility increases along with the charge carrier density.

\subsubsection{Doping of conjugated polymers}

In its pristine state, a conjugated polymer may be intrinsically semiconducting. The low number of thermally excited charge carriers means intrinsic conjugated polymers have a relatively large band gap (typically between 2 and $3 \mathrm{eV}$ ). It is possible to boost the number of charge carriers in these systems through oxidation or reduction. The oxidation/reduction of a conjugated polymer involves the addition/removal of electrons to/from the polymer, in a process called $p$-doping/n-doping. ${ }^{6}$ The term doping is used by analogy with the doping of inorganic semiconductors. Doping increases the number of charge carriers (e.g. solitons, polarons or

\footnotetext{
${ }^{6}$ Because of the prevalence of oxygen on Earth, n-doped polymers are rare as most of them undergo a spontaneous oxidization when exposed to the athmosphere.
} 
bipolarons). Oppositely charged ions compensate for these newly created charges in order to maintain electroneutrality on the polymer. Doping may be achieved chemically as well as electrochemically.

Chemical doping transfers electrons between a dopant molecule and the conjugated polymer. In the case of p-doping/n-doping, oxidation/reduction of the conjugated polymer is achieved by ensuring that it has a LUMO/HOMO lower/higher than the HOMO/LUMO of the dopant. In the case of chemical doping, the dopant molecule itself compensates for the charge created on the polymer. The ability to tune the electrical conductivity through chemical doping is often exploited in sensing applications.

Electrochemical doping is achieved by using the conjugated polymer as the working electrode in a two electrode setup. An auxiliary electrode, along with the working electrode, is placed in an electrolytic solution. By applying a potential difference between the two electrodes, positive/negative charges start to appear in the polymer as it undergoes oxidation/reduction. In this case, negative/positive ions from the electrolyte compensate for the polymer's newly formed positive/negative charges. Electrochemical doping is central to a variety of organic electronics applications, from logic gates to displays.

\subsubsection{Electrochromism in conjugated polymers}

Electrochromic materials exhibit the property of "change, evocation, or bleaching of color" as a result of undergoing a redox process (or by a sufficient electrochemical potential).[18] Electrochromic materials may be inorganic (e.g. oxides of transition metals) or organic (e.g. resonance stabilized aromatic molecules).[18] Since the birth of electrochromic technology four decades ago, these materials have attracted considerable attention for their potential in display applications.[19] Electronic paper is an umbrella term that encompasses several technologies, including electrochromic displays.

Among the most widely studied electrochromic materials are $\mathrm{WO}_{3}, \mathrm{MoO}_{3}, \mathrm{TiO}_{2}, \mathrm{Ta}_{2} \mathrm{O}_{5}, \mathrm{~V}_{2} \mathrm{O}_{5}$ and $\mathrm{Nb}_{2} \mathrm{O}_{5}$. [20, 21] Interest in electrochromic displays based on conjugated polymers appeared much later, which explains why the latter are conspicuously absent from commercial applications. ${ }^{7}$ In contrast with metal oxides, electrochromic materials based on conjugated polymers offer higher

\footnotetext{
${ }^{7}$ The Boeing 787's windows are equipped with electrochromic metal oxide windows to replace the conventional plastic pull-down shades.
} 


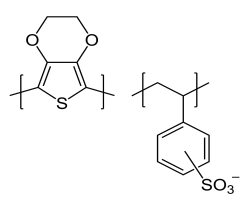

(a) Chemical structure of PEDOT:PSS.

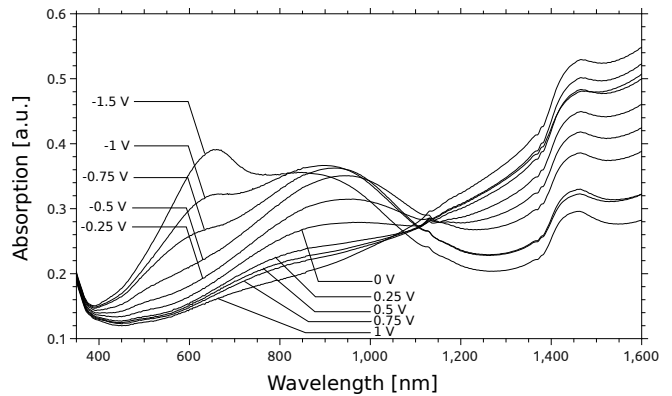

(b) Optical absorption spectrum of reduced/oxidized PEDOT:PSS.

Figure 2.12: Electrochromism basics.

contrast, are cheaper, more durable, and easier to synthesize as well as to process. [21]

As explained in the previous section, doping a conjugated polymer shrinks its band gap. Provided the band gap of the doped state, undoped state, or both, falls within the range of energies of visible light ${ }^{8}$, the polymer will exhibit a color change upon doping.

A conducting polymer commonly explored in electrochromic display settings is poly(3,4-ethylene-dioxythiophene) — PEDOT - doped with poly(styrene-sulfonate) - PSS. Figure 2.12(a) shows a PEDOT (left) and a PSS (right) molecule. This system is referred to as PEDOT:PSS and, along with viologen derivatives, is the most commonly used organic electrochromic material.[22] Figure 2.12(b) shows the absorption spectrum of PEDOT:PSS at different voltages that correspond to different redox levels. When PEDOT:PSS is reduced (i.e. the negative voltages in figure $2.12(\mathrm{~b})$ ), it begins to absorb significantly more in the visible spectrum and turns into a dark shade of blue. On the other hand, when oxidized (i.e. positive voltages), the PEDOT:PSS absorbs in the IR region which turns it into a transparent light blue. It should be noted that a minor tint of blue typically becomes visible in the oxidized state as well, and is due to the layer thickness required by most electrochromic applications.

\footnotetext{
${ }^{8} \mathrm{~A} 400-700 \mathrm{~nm}$ wavelength corresponds to $2-3 \mathrm{eV}$ in terms of energy.
} 


\section{$2.2 \quad$ Polyelectrolytes}

\subsubsection{Electrolytes}

An electrolyte is any substance that contains free moving ionized/dissociated species. The movement of the latter is responsible for the electrical conductivity of electrolytes. In other words, the ions move when subjected to an electric field. When sodium chloride $(\mathrm{NaCl})$ is in contact with water, the dissolved sodium and chloride atoms become ionized. Along with electrodes, electrolytes are a central component for building electrochemical cells. Electrolytes are also critical for the physiology of all (known) higher lifeforms.

As long as no ion-pairs are formed, the conductivity of an electrolyte increases with the ion concentration.[23] Electrolytes can be strong or weak depending on their degree of dissociation. Besides electrolytic solutions, a variety of other substances fall in the electrolyte category:

- Ionic liquids are salts that exist in their liquid form under $100{ }^{\circ} \mathrm{C}$. This class of electrolytes are non-volatile, have a high ionic conductivity (up to $100 \mathrm{mS} / \mathrm{cm}$ ), and a large electrochemical potential window.[24]

- Ion gels are ionic liquids which have been gelled in a polymer matrix (typically, a block copolymer).[25]

- Polymer electrolytes consist of a salt dissolved in a polymer matrix (e.g. $\mathrm{NaCl}$ in $\mathrm{PEO}$ ). The ionic conductivity of these systems is around $10^{-4} \mathrm{~S} / \mathrm{cm} .[26]$

- Polyelectrolytes are charged polymers with free moving counterions. The ionic conductivity of polyelectrolytes is around $10^{-3} \mathrm{~S} / \mathrm{cm} .[27]$

- Poly(ionic liquid)s are a class of polyelectrolytes which contain an ionic liquid species in each monomer repeating unit. The conductivity for poly(ionic liquid) systems has been reported as reaching $10^{-4} \mathrm{~S} / \mathrm{cm}$.[28]

\subsubsection{Polyelectrolytes}

Polyelectrolytes are charged polymers whose repeat units contain an electrolyte group which spontaneously dissociates in a polar solvent. Polyanions refer to polyelectrolytes with a negatively charged 
<smiles>CCC(C)c1ccc(S(=O)(=O)O)cc1</smiles>

(a) PSS:Na.

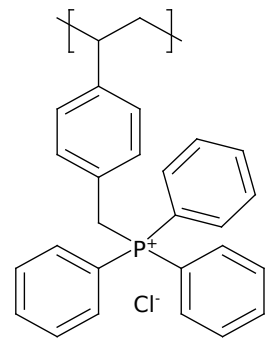

(b) $\mathrm{PVBPPh}_{3}: \mathrm{Cl}$.

Figure 2.13: Polyelectrolytes.

backbone and positively charged counter-ions - e.g. poly(styrene sulphonic acid) sodium [PSS:Na] shown in figure 2.13(a). Correspondingly, a polycation has a positively charged backbone and negative counter-ions - e.g. the polyphosphonium chloride $\left[\mathrm{PVBPPh}_{3}: \mathrm{Cl}\right]$ in figure 2.13(b). Polyelectrolytes are used to produce detergents, paints, shampoos, lotions and processed food.[29] Thanks to their printability, polyelectrolytes are of particular interest to the production of organic electronic components. [25, 30]

The ions which are bound to the polymer chain are called coions while the mobile ions are referred to as counter-ions.

\subsubsection{Charge transport in polyelectrolytes}

The motion of ions in electrolytes is typically governed by one of the following processes: diffusion and migration.

If a concentration gradient $\Delta c$ exists in an electrolyte, ions will diffuse from the high concentration region to the low one. The diffusional flux $(J)$ of ions resulting from a concentration gradient $\Delta \mathrm{c}$ is described by Fick's first law as follows: ${ }^{9}$

$$
J=-D \Delta c
$$

Migration occurs when the motion of ions is due to the presence of an electric field across an electrolyte. Interestingly, ion migration almost inevitably results in a concentration gradient which, in turn, subjects the ions to diffusion. As anions and cations typically diffuse at different rates, this asymmetry induces an electric field

\footnotetext{
${ }^{9}$ Where $D$ is the diffusion coefficient, and is proportional to both the viscosity of the electrolyte and the size of the solvated ion.
} 
across the electrolyte resulting in migration. The total flux of ions is described by the Nerst-Planck equation:

$$
J=-D \Delta c+\frac{z F}{R T} D c \Delta \phi
$$

Where $z$ is the charge $(z=-1$ for anions and $z=+1$ for cations), $c$ is the concentration, $F$ is Faraday's constant, $R$ is the gas constant and $T$ the temperature.

In polyelectrolytes, the diffusion coefficient is very dependent on the mobility of the polymer chains. The mobility of the latter increases with the presence of solvents and decreases with the degree of crystallinity.

\subsubsection{Ionic selectivity}

Ion exchange membranes are semipermeable membranes used to separate, concentrate or exclude ions from a liquid electrolyte.[31] A polyelectrolyte that is kept in a solid form by cross-linking or by hydrophobic forces is an ion exchange membrane.[32] In this phase, the polymer backbone is - mostly - immobile and only ions of one polarity may move freely.

If an ion exchange membrane is surrounded by an electrolyte, ions which are of the same polarity as the membrane's co-ions may be electrostatically hindered from entering the membrane by a process called Donnan exclusion. This holds as long as the electrolyte concentration remains well below the concentration of co-ions in the membrane. This effect is also due to the fact that one of the ionic functionalities in ion exchange membranes is immobilized. The ionic selectivity of polyelectrolytes has been exploited to make ionic bipolar junction transistors and diodes. [33, 34]

Figure 2.14 shows an electrolyte on both sides of a cation exchange membrane. When no potential is applied across the system, the distribution of ions in the electrolyte is random (a). As the concentration of the membrane's co-ions becomes higher than that of the electrolyte's ions, the membrane electrostatically repels the ions of the same polarity as its fixed groups. However, the counterions are able to migrate through the membrane. This is due to the ability of the (mobile) counter-ions to redistribute in a polarized membrane to accommodate the flow of ions from the electrolyte. 
a)

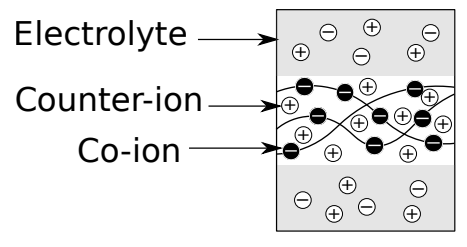

b)

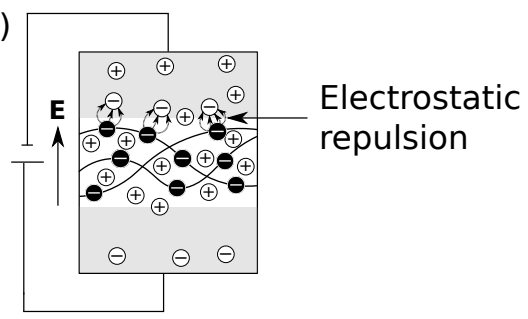

Figure 2.14: Ion migration (a) — and lack thereof (b) - across an ion exchange membrane.

\subsection{Cellulose}

Cellulose is the most abundant organic compound on the planet and is used to produce paper by dewatering a dilute suspension of cellulose fibers followed by pressing and baking. [35, 36] Up until a couple of decades back, paper has inarguably been the most instrumental tool for the dissemination (and conservation) of knowledge. ${ }^{10}$ In fact, the first description of paper produced from plant fibers dates back to the year $450 \mathrm{CE}$ in the Book of the Later Han.[37] The chemical structure of cellulose is depicted in figure 2.15. This carbohydrate polymer consists of $\beta$-D-glucopyranose repeating units which are covalently bound through acetal functions ( $\beta$-1,4-glucan).[38]. The chemical formula of cellulose is $\left(\mathrm{C}_{6} \mathrm{H}_{10} \mathrm{O}_{5}\right)_{n}$, with $n$ between 10,000 and 15,000.[39] Cellulose is symbolically tied to the polymer revolution as it was used to produce the first thermoplastic (i.e. celluloid).[40] However, celluloid is highly flammable and its (laborious) production has been decimated after the International Table Tennis Federation switched to polystyrene balls in 2014 .

This almost inexhaustible raw material is inextricably intertwined with the concept of printed electronics. Several techniques have already been demonstrated to decrease the large surface roughness and high porosity of paper as a substrate for electronic components.[41] At 0.1 cent per square decimeter, paper is indeed substantially cheaper than alternative substrates (compare with polyethylene terephthalate: $2 \mathrm{c} / \mathrm{dm}^{2}$, and polyimide: $\left.30 \mathrm{c} / \mathrm{dm}^{2}\right) .[36]$ Moreover, modern paper machines may be up to 200 meters long

\footnotetext{
10 "Writing without a medium is unthinkable. We owe to paper our ability to live as people and leave an honest accounting." — G. Plinius, Naturalis Historia, $79 \mathrm{CE}$
} 


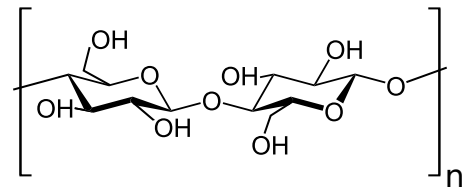

Figure 2.15: Chemical structure of cellulose.

and, are capable of processing (10-meters-wide) sheets at neckbreaking speeds of more than $100 \mathrm{~km} / \mathrm{h}$.[37] Electronic circuitry produced on paper is likely to have a very competitive cost per unit.

\subsubsection{Manufacturing}

Cellulose may be extracted from plants, bacteria as well as algae. Most often, the starting material is a wood log that undergoes debarking ${ }^{11}$ mechanically or hydraulically. This $\log$ is then transformed into a water suspension of cellulose, hemicellulose and lignin. Thereafter, the cellulose is separated from the other fibers through a process called pulping which may be mechanical, chemical or semimechanical. Chemical pulping accounts for the majority of global pulp production as it produces the highest quality cellulose.[42] In contrast, mechanical pulping breaks down the fibers and does not remove nearly as much of the lignin.

\subsubsection{Cellulose nanofibers}

Delaminating cellulose fibers in a high shear homogenizer produces cellulose nanofibers (CNF) with a high aspect ratio (5-20 nm x 1000-5000 $\mathrm{\mu m})$. Homogenization is achieved by a piston which forces the suspension through a miniscule channel under a pressure of up to 2,000 MPa.[43] Other techniques to produce CNF include microfluidization, ultrasonication and micro-grinding. Figure 2.16 shows an AFM image of a CNF film (false color scale not shown).

CNF films exhibit excellent mechanical properties due to stronger fibrils and superior interfibril adhesion. Also, the interstitial voids between the fibers are smaller in size and more homogeneously distributed. CNF films have been shown to have a Young's modulus above $10 \mathrm{GPa}$ and a tensile strength above $200 \mathrm{MPa}$.[44] Therefore,

\footnotetext{
${ }^{11}$ Not to be confused with: 1 . the action of leaving a ship and 2. a dog's ventriculocordectomy.
} 


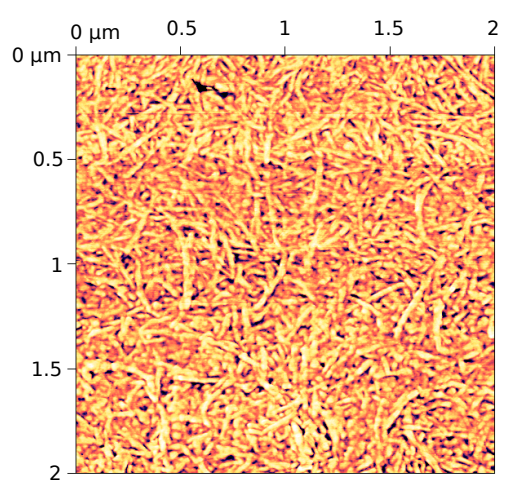

Figure 2.16: AFM image of nanofibrillated cellulose.

CNF are good candidates to produce dry yet flexible composites with improved mechanical integrity. Several techniques may be employed to produce these composites (viz. solvent casting, melt mixing, in-situ polymerization and electrospinning).[43]. Moreover, CNF may be composited with other polymers as well as a wide range of micro- or nano-particles.[45, 46]

Owing to its biocompatibility, biodegradability and low toxicity, CNF have also been touted as having "great potential for the breakthrough of a novel generation of biomedical materials".[47] Indeed, CNF has been investigated to replace the nucleus pulposus (inner core of the vertebral disc) as well as to stabilize and control the delivery of protein-coated drugs. $[48,49]$. 


\section{Chapter 3}

\section{Devices}

\subsection{Capacitors}

Capacitors are passive two-terminal energy-storage devices which are fundamental to the field of electronics. Unlike resistors which allow electrons to flow through them in proportion to the voltage drop $(I=V / R)$, capacitors oppose changes in voltage $\left(I=C \frac{d U}{d t}\right)$. But their usefulness goes beyond exploiting their electrical reactance in electrical circuits. Capacitors and batteries have indeed been assigned equal importance when considering the energy storage systems of tomorrow.[50]

In this section, the conventional capacitor, the electrostatic double layer capacitor as well as the electrochemical pseudocapacitor are briefly described.

\subsubsection{Solid dielectric capacitor}

\subsubsection{Parallel-plate capacitor}

The conventional capacitor consists of two conducting plates separated by a dielectric material. In the standard model, said plates are parallel to each other. A solid dielectric capacitor stores energy electrostatically. In other words, the dielectric capacitor relies on purely non-faradaic processes.

The ability of a molecule to become polarized under an external electric field (known as permittivity $\varepsilon$ ) is the material property which determines the capacitance of a device. By definition, the relative permittivity of air is 1 . Capacitors can be made with a 
a)

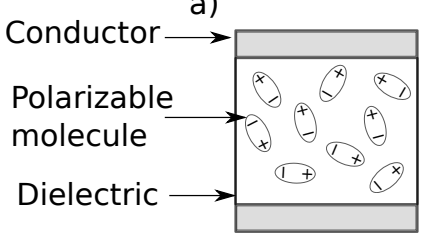

b)

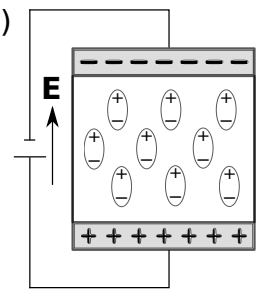

c)

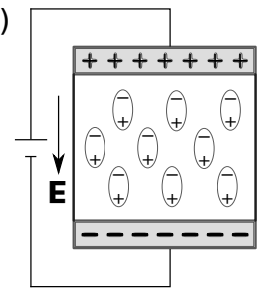

Figure 3.1: Polarization of a capacitor with permanent dipole moment dielectric. In the absence of an electric field (a) the molecules are randomly oriented. When an electric field is applied (b and c), the molecules align as a function of its polarity.

variety of dielectric materials such as glass, paper, polystyrene, PTFE (Teflon) or even air. The conducting plates are typically made of metal sheets.

The tendency of an atom to attract electrons - electronegativity - depends on its atomic number and on the distance of the valence electrons from the nucleus. A molecule composed of atoms with different electronegativities ends up carrying a net charge, and is said to have a permanent dipole moment. Figure 3.1 shows a simplified illustration of what happens in a capacitor with a dielectric that contains permanent dipoles.

The capacitance of a parallel-plate capacitor can be calculated by the following equation:

$$
C=\frac{\varepsilon A}{d}
$$

The permittivity is described by the quantity $\varepsilon$. $d$ is the distance between the plates and $\mathrm{A}$ the area of the latter.

Similarly, the capacitance per area $\mathrm{C}_{\mathrm{i}}$ is: $C_{i}=\frac{\varepsilon}{d}$

\subsubsection{Metal-insulator-semiconductor capacitor}

Intercalating a semiconducting layer inside the structure described above results in a metal-insulator-semiconductor capacitor (MIS). ${ }^{1}$ Figure 3.2 shows the basic structure of a MIS capacitor. The capacitance of a MIS capacitor is calculated in the same way as that of a parallel-plate one.

\footnotetext{
${ }^{1}$ Also called metal-oxide-semiconductor (MOS) as the insulating layer has historically been made of an oxide material.
} 


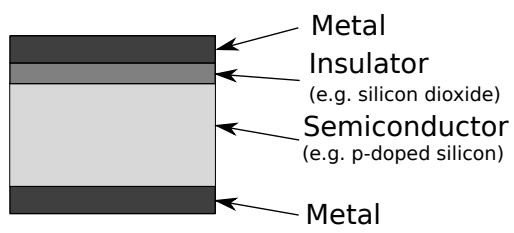

Figure 3.2: Illustration of a metal-insulator-semiconductor capacitor.

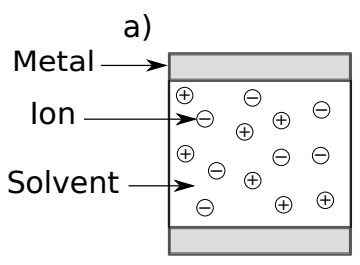

b)

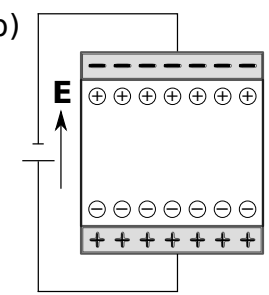

c)

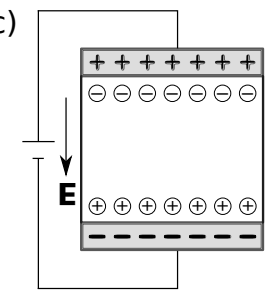

Figure 3.3: Polarization of ions creates EDLs in a parallel plate capacitor. In the absence of an electric field (a) the ions are randomly located. When an electric field is applied (b and c), the ions migrate towards the oppositely charged electrode.

\subsubsection{Electrostatic double layer capacitor}

Unlike dielectric materials which are electrically insulating, an electrolyte contains ions that may move if an electric field is applied to it. So, assuming that the conducting electrodes are electrochemically inert, replacing the dielectric with an electrolyte is a way to construct an electrostatic double layer capacitor. When a charged metal electrode comes in contact with an electrolyte, the ions move to compensate for the charges on the electrode. Figure 3.3 shows an electrostatic double layer capacitor (EDLC) with zero-bias across it (a), then the creation of electric double layers (EDLs) as a potential difference occurs at the metal/electrolyte interface (b and c). In this type of capacitor, the electrons involved in the charging/discharging come from the delocalized conduction-band of the metal.[51]

The charges on the metal electrode along with the thin layer of ions in their vicinity form an EDL. There are two EDLs in an electrostatic double layer capacitor (such as the one depicted in figure 3.3).

The Goüy-Chapman-Stern model is typically used to describe 


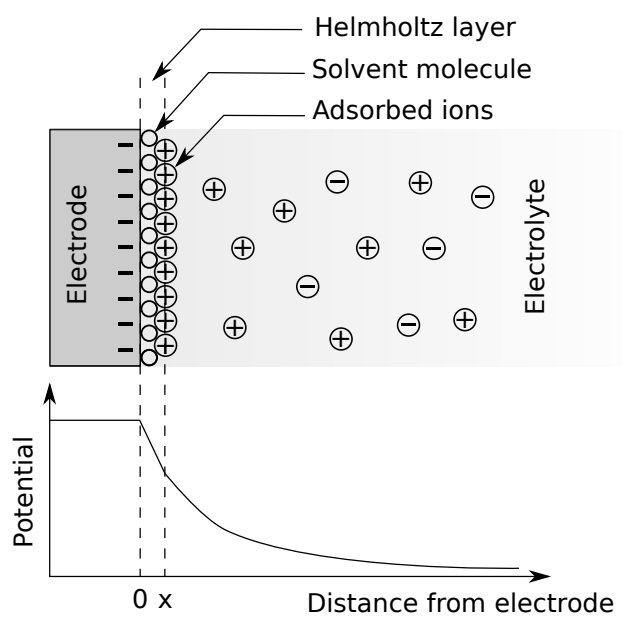

Figure 3.4: Ionic distribution in the Goüy-Chapman-Stern model with the electric potential profile at a metal/electrolyte interface.

the charge distribution in an EDL.[52] In this model (illustrated in figure 3.4), the adsorbed solvent molecules and solvated ions in the vicinity of the electrode represent the Helmholtz layer. The thickness of this layer (x) is of the order of the ångström (radius of the involved ions) and is analogous to an extremely thin parallel plate capacitor with an enormous capacitance per area that reaches into the hundreds of $\mu \mathrm{F} \mathrm{cm} \mathrm{cm}^{-2}$.[53]

Adjacent to the Helmholtz layer is the diffuse layer which extends towards the bulk of the electrolyte. In contrast to the Helmholtz layer which consists of ions of opposite polarity to that of the electrode, the diffuse layer contains ions of both polarities. The potential drop across this layer is exponential with a capacitance per area that may reach tens of $\mu \mathrm{F} \mathrm{cm}^{-2}$. [54, 55]

Figure 3.4 shows the electric potential profile in the GoüyChapman-Stern model. The large ion density in the thin Helmholtz layer causes a steep (and linear) potential drop. However, the potential drop is more gradual (and exponential) in the aptly-named diffuse layer.[51]

In an EDLC, virtually all the potential drop occurs at the two interfaces shown in figure 3.3. These interfaces may be viewed as two capacitors in series, which means that the total capacitance is limited by the smallest of these two interfacial capacitances. The thickness of the electrolyte does not negatively affect 
a)

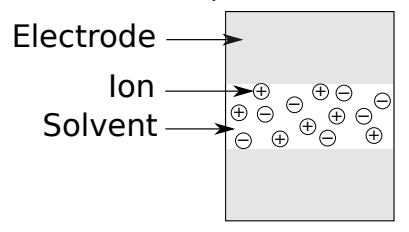

b)

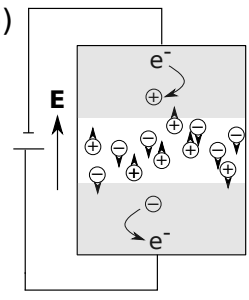

c)

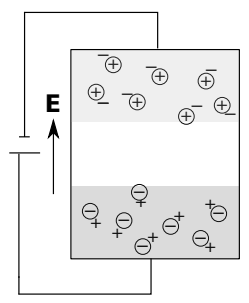

Figure 3.5: Randomly distributed ions in the electrolyte (a) start moving under the influence of an electric field (b). Thereafter, oxidation/reduction takes place at the anode/cathode.

the capacitance.[56]

\subsubsection{Electrochemical pseudocapacitor}

Electrochemical pseudocapacitors, by opposition to the electrostatic capacitors described above, involve a phase change in the reactant species which make up the electrodes. These type of capacitors offer several advantages such as an exceptional stability ${ }^{2}$ and a large power density over other energy storage elements. [50] Being a faradaic process, the storage of charges involves a transfer of electrons which are shuttled towards (or originate from) orbitals of the redox anode (or cathode) reagent.[51] The electrodes are typically made from oxides and sulfides of transition metals (e.g. $\mathrm{RuO}_{2}, \mathrm{IrO}_{2}, \mathrm{TiS}_{2}$ ) [57], but there is a long-standing interest in using conducting polymers.[58] In an electrochemical capacitor, the ions move past the double-layer, and penetrate into the - electrochemically active - electrode (see figure 3.5). This process is accompanied by a transfer of electrons which are shuttled towards (originate from) orbitals of the anode (cathode) reagent and results in an oxidative (reductive) reaction.

In practice, the total capacitance of a pseudocapacitor has an electrostatic component (between 5 to 10\%). And interestingly, up to $5 \%$ of the capacitance in an electrostatic double layer capacitors with carbon electrodes is pseudocapacitive ${ }^{3}$.[51]

The phase change in the electrode in (the simplistic) figure 3.5 is indicated by a nuance in color.

\footnotetext{
${ }^{2}$ In case of a central power supply failure, the Airbus A380's 16 emergency doors rely on banks of electrochemical supercapacitors to be operated.

${ }^{3}$ This pseudocapacitance is due to the faradaic reactivity of oxygencontaining functional group present at the surface of carbon electrodes.
} 


\subsection{Organic electrochromic display}

As described in section 2.1.7, certain polymers (e.g. PEDOT:PSS) change color when undergoing a redox reaction. Organic electrochromic displays have the same structure (and mode of operation) as the electrochemical capacitors described in the section above. Two electrochromic polymer electrodes coupled with an ionically-conducting solid electrolyte (i.e. polyelectrolyte) is the most basic structure of an organic electrochromic display. These devices are commercially attractive since all the involved components are printable. Moreover, such devices typically display shorter switching times than their inorganic counterpart.

Figure 3.6 shows the basic structure of an organic electrochromic display. In this case, the electrodes consist of PEDOT:PSS and the electrolyte is a polyanion. As long as the device is in an open circuit mode (a), the PEDOT:PSS electrodes remain in their pristine state. When a potential is applied across the device (b and c), the mobile ions (cations) in the polyelectrolyte migrate towards the oppositely charged electrode. The latter is reduced and switches color from light to deep blue as indicated by the color code (e). The current versus voltage characteristics of the display (d) is governed by the charging/discharging of the electrodes similar to the electrochemical capacitor previously described.

It should be noted that the oxidized electrode also changes color and turns light blue, negatively affecting the contrast of a vertical device that operates in transmission mode. Several schemes were demonstrated to overcome this issue. [59, 60, 34]

Figure 3.7 shows photographs of a $100 \mathrm{~nm}$ film of PEDOT:PSS in oxidized - 3.7(a) - and reduced - 3.7(b) - form in a device employing an opaque electrolyte layer.[60] The opacity of the latter changes the device operation from transmissive to reflective mode.

\subsection{Bipolar membrane diode}

\subsubsection{Diode}

A diode (see figure 3.3.1) is a two-terminal - most often passive ${ }^{4}$ - electronic component. The main function of a diode is to block the current in one direction (the reverse direction) while allowing it to flow in the other (the forward direction). This feature, typically

\footnotetext{
${ }^{4}$ Certain diodes which exhibit a negative differential resistance can be categorized as active.
} 
a)

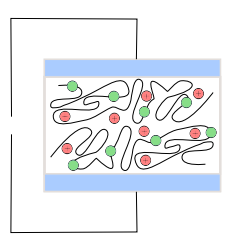

d)

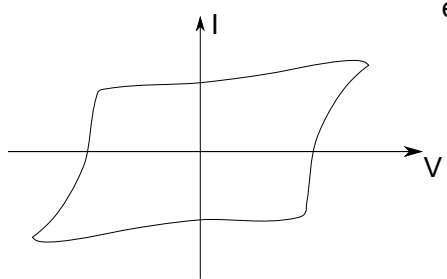

b)

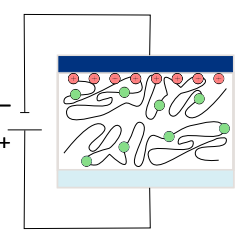

e)

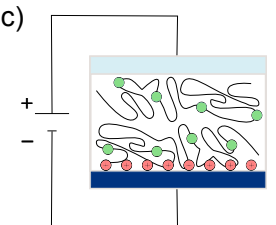

PEDOT:PSS

Anion selective membrane

Cation

Oxidized PEDOT:PSS

Reduced PEDOT:PSS

Figure 3.6: Basic operation of an organic electrochromic display $(\mathrm{a}-\mathrm{c})$, with I-V curve $(\mathrm{d})$ and color code $(\mathrm{e})$

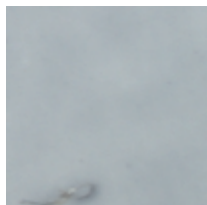

(a) Oxidized (at $3 \mathrm{~V})$.

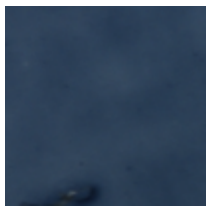

(b) Reduced (at $-3 \mathrm{~V})$.

Figure 3.7: Photographs of PEDOT:PSS in the oxidized and reduced state. 


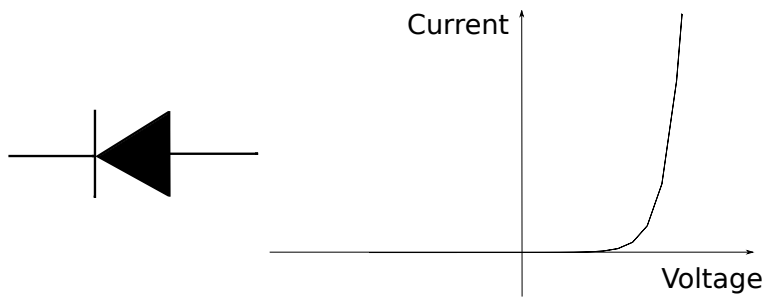

(a) Electronic sym- (b) I-V characteristics of a diode. bol.

Figure 3.8: Diode.

called current rectification, may be exploited to convert alternating current (e.g. household electricity, radio signals) into direct current. Unlike a resistor or a capacitor, a diode is a nonlinear component as it does not obey Ohm's law.[61] Instead, a diode's current is an exponential function of the voltage across it. Figure 3.8 shows the $\mathrm{I}-\mathrm{V}$ characteristics of a typical silicon PIN diode.

\subsubsection{Bipolar membrane}

A bipolar membrane (BM) consists of two selective membranes of opposite polarity (a cation-exchange membrane and an anionexchange membrane) in contact with each other. Figure 3.9 depicts the movements of ions inside a bipolar membrane. When no bias is applied (a), the mobile cations and anions are randomly distributed within their respective membrane as counter-ions, with few mobile co-ions present. When a potential is applied across the $\mathrm{BM}$, the counter-ions start to move towards (or away from, depending on the polarity) the junction at which the two membranes make contact. If the anion-selective membrane is positively biased, the counter-ions (anions) of that particular membrane will migrate towards the junction. Similarly, the counter-ions (cations) in the cation-selective membrane also move towards the junction and, as long as both anions and cations may be replenished (e.g. from adjacent electrolytes) an ionic current flow can be sustained. This - forward bias - regime is illustrated on the right-hand side of figure 3.9(d). However, if the polarity is reversed, the mobile ions migrate away from the junction, and an ion-poor volume is formed (a depletion region). This mode of operation - reverse bias - is depicted in figure 3.9(c) by the non-shaded region. In the reverse 
bias the potential drop across the junction is increased due to the low conductivity of the depleted region. At a certain potential, the confinement of a large electric field within the junction can cause the dissociation of water molecules to occur at a higher rate. This produces protons and hydroxide ions which start to dominate the ionic current by moving towards the cathode and anode, respectively. The water-dissociation regime is shown in figure 3.9(b).

\subsection{Transistors}

\subsubsection{Overview}

A transistor is a three-terminal active device used for the switching and amplification of electronic signals. It is the fundamental component of every electronic circuit.[61] As billions of them are crammed into a single chip $^{5}$, transistors happen to be the most abundant and pervasive discrete electronic component around. Although Shockley, Bardeen, and Brattain were awarded the 1956 Nobel Prize in Physics for the "discovery of the transistor effect", earlier work by Lilienfeld and Heil preceded their point-contact transistor.[62, 63]

When operating as an amplifier, a transistor takes the small electric current applied to the input terminal and produces a much larger current at the output. It is this power gain which distguinshes transistors from voltage-amplifying passive components such as step-up transformers. This property of power amplification in a solid-state device enabled the mass adoption of radio transmission technology which had, up until the 1940s, been constrained to using the bulkier, costlier and more fragile vacuum tubes. The miniaturization of transistors also brought about the portable transistor radio revolution.

Yet, transistors had an even greater impact when used as switches as they enabled digital computing. Indeed, a transistor switch is a considerably more efficient, compact, cheap and reliable way to construct logic gates (i.e. the building blocks of microcontrollers and microprocessors) than the clunky electromechanical relays or vacuum tubes. Moreover, transistors can switch at blistering subnanosecond speeds.

\footnotetext{
${ }^{5} \mathrm{~A}$ single Intel Xeon Haswell-EP CPU chip contains over 5.5 billion transistors.
} 
a)

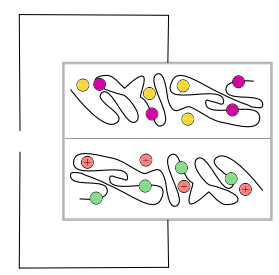

e) Anion

Cation selective membrane

Anion selective membrane

Cation

\begin{tabular}{|l|}
\hline Water \\
\hline Proton \\
\hline Hydroxide \\
\hline
\end{tabular}

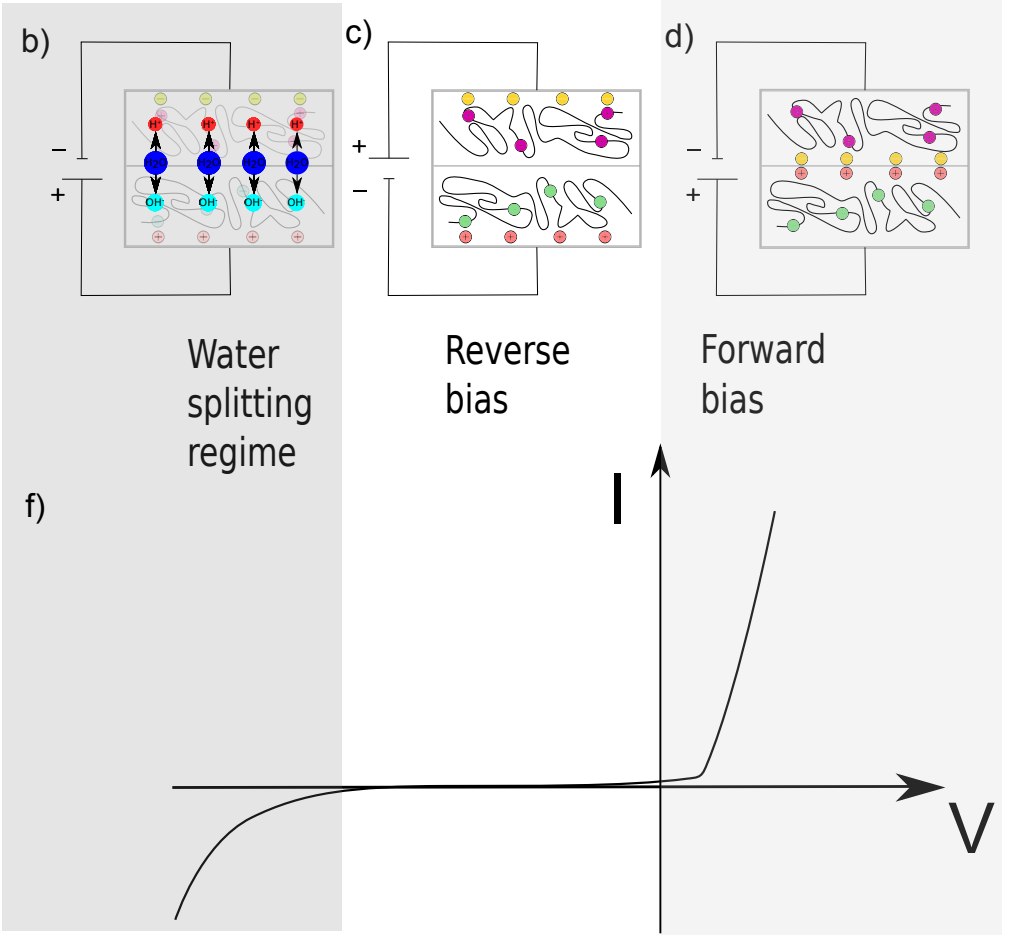

Figure 3.9: Ion migration in bipolar membrane and I-V curve in forward bias (d), reverse bias (c) and water-dissociation mode (b). 


\subsubsection{Bipolar junction transistor}

Bipolar junction transistors (BJT) comprise two semiconducting $\mathrm{p}-\mathrm{n}$ junctions. The $p$ region is made by doping silicon with an acceptor atom such as boron. Boron atoms, which only contain three valence electrons, substitute some silicon atoms in the crystal lattice. As this happens, a bond remains unsatisfied on an adjacent tetravalent silicon atom. This lack of an electron is called a hole and has the ability to move around the lattice in the following fashion: when an electron from a neighboring bond moves in to fill the hole, it leaves a hole behind. Similarly, creating a $n$ region in silicon involves doping with a donor atom (e.g. phosphorous) which provides extra electrons.

The bipolar refers to the fact that both electrons and holes are involved in the operation of BJTs. Figure 3.10 shows the schematic symbol (a) and device operation (b) of an NPN-type BJT. For several decades, BJTs reigned supreme on the transistor technology. It should be noted that BJTs are available in two polarities: NPN and PNP. The three terminals are termed base (B), collector (C) and emitter (E). As the B-E junction is biased with a sufficient voltage, electrons from the emitter start getting injected into the base. From there, the electrons start to diffuse towards the collector.

\subsubsection{Field-effect transistor}

\subsubsection{Basics}

A field-effect transistor (FET) is a transistor where the channel's conductance (i.e. the ability of electrons to flow between the source and the drain) is modulated by the application of an external electric field. An important feature of FETs is that the gate terminal draws no current in operation. More importantly, FETs have a very high input resistance (up to $10^{14} \Omega$ ), and a very low output resistance. Unlike BJTs, FETs contain no forward-biased junctions. But like BJTs, FETs come in two polarities: n-type and p-type transistors, where the carriers are electrons and holes, respectively. Transistors which are able to function as both n- and p-type devices are (aptly) called ambipolar. For simplicity, the following sections will focus on n-type FETs. Electrons also happen to display better mobility and carrier lifetime. [61]

Figure 3.11 shows the typical symbol used for an FET. By analogy to the BJT's emitter, collector and base, the FET terminals are 
a)

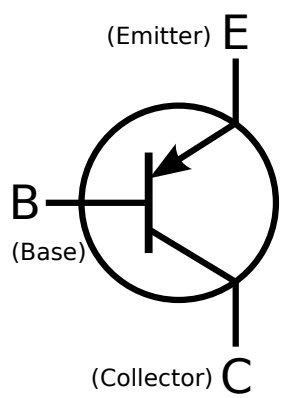

b)

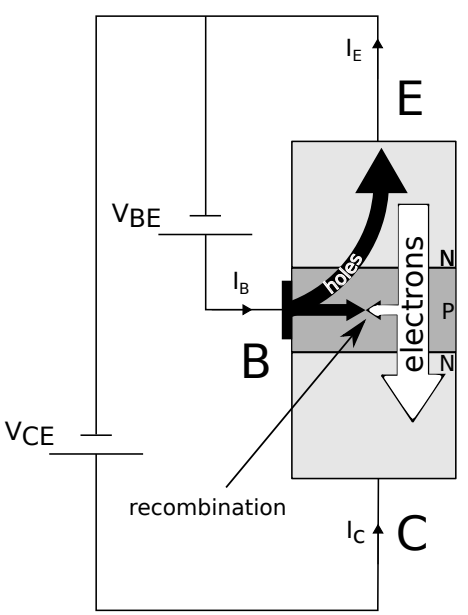

Figure 3.10: NPN bipolar junction transistor, with reverse-biased B-C junction and forward-biased B-E junction. Schematic symbol (a) and device structure (b).

source $(\mathrm{S})$, drain $(\mathrm{D})$ and gate $(\mathrm{G})$ terminals. The semiconducting region between $\mathrm{S}$ and $\mathrm{D}$ is called the channel.

Electrons enter the channel through the source and leave it through the drain terminal. The potential applied between the gate and drain modulates the flow of electrons through the channel.

The structure of an FET is depicted in figure 3.12. The substrate on which the FET is built is omitted for simplicity. The channel in an FET is typically constrained to a thin semiconducting layer closest to the insulator.

Depending on the initial doping of the channel, FETs may operate in: enhancement or depletion mode. The channel in an enhancement FET is non-conducting at zero (or negative) gate bias. A depletion FET has a doped channel which allows current to flow between source and drain even at zero gate bias. To cut off the drain current, a sufficient potential (threshold voltage, $\mathrm{V}_{\mathrm{th}}$ ) has to be applied between the gate and source terminals. Figure 3.13 depicts the distinction between enhancement and depletion transistors. Note that the y-axis represents a logarithmic plot of the drain current.

For an enhancement-mode FET, the voltage that needs to be applied to the gate for it to become conducting is also the threshold voltage $\left(\mathrm{V}_{\mathrm{th}}\right)$. The gate-insulator-semiconductor structure is simi- 


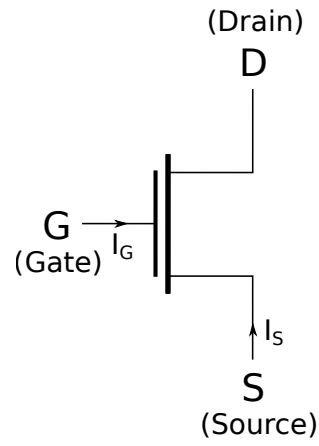

Figure 3.11: Field-effect transistor symbol.

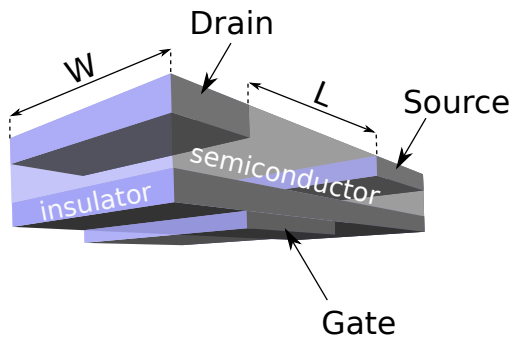

Figure 3.12: Field-effect transistor structure.

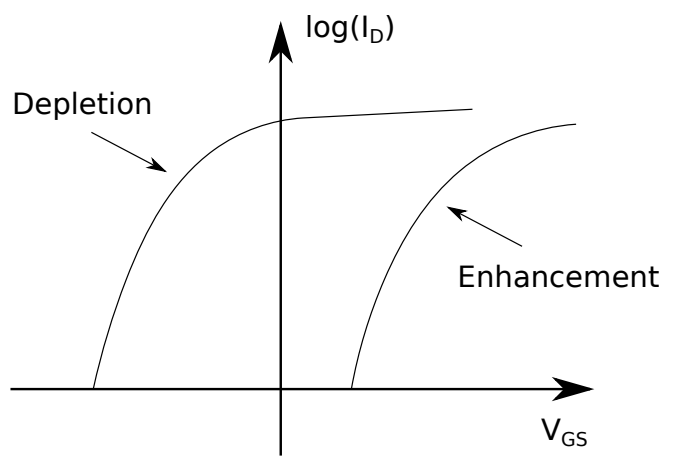

Figure 3.13: Transfer curves of two FETs to illustrate the distinction between enhancement-mode and depletion-mode devices. 
lar to that of MIS capacitors described in section 3.1.1.2 (compare figure 3.2 and 3.12).

The charge density at a given point $\chi$ along the channel is shown in equation 3.2 .

$$
Q(\chi)=C_{i}\left(V_{G S}-V_{t h}-V_{\chi}\right)
$$

Figure 3.14 shows the charge distribution in an FET and the corresponding current-voltage characteristics. For the channel to be conducting, the gate bias (relative to source) has to be superior to $\mathrm{V}_{\text {th }}$. As a voltage is applied to the drain, the amount of charges flowing in the transistor channel increases. This flow encounters a constant electrical resistance as long as $V_{D S} \ll V_{G S}-V_{t h}$. This linear regime is depicted in figure $3.14(\mathrm{a})$.

The drain current at a given point $\chi$ along the channel may be described by equation 3.3 , where $\mu$ is the charge carrier mobility. $\mathrm{E}(\chi)$ is the electric field at a position $\chi$ and is equal to $\frac{\partial V(\chi)}{\partial(\chi)}$.

$$
I_{D}(\chi)=W \mu Q(\chi) E(\chi)
$$

In the linear regime, where $V_{D S} \ll V_{G S}-V_{t h}$, the drain current may be approximated by equation 3.4.

$$
I_{D(\text { lin })}=\frac{W \mu C_{i}}{L}\left(V_{G S}-V_{t h}\right) V_{D S}
$$

The field-effect mobility in the linear regime is derived from equation 3.4, resulting in equation 3.5.

$$
\mu_{l i n}=\frac{L}{W C_{i} V_{D S}} \frac{\partial I_{D(l i n)}}{\partial V_{G S}}
$$

At saturation, where $V_{D S}=V_{G S}-V_{t h}$, equation 3.2 yields the following expression for the drain current.

$$
I_{D(s a t)}=\frac{W \mu C_{i}}{2 L}\left(V_{G S}-V_{t h}\right)^{2}
$$

In this regime, charges are constricted at the drain electrode as illustrated in figure 3.14(b). This state is called pinch-off and represents the onset of the saturation regime (figure 3.14(c)).

Applying a derivative to equation 3.6 gives the field-effect mobility at saturation.

$$
\mu_{s a t}=\frac{2 L}{W C_{i}}\left(\frac{\partial \sqrt{I_{D(s a t)}}}{\partial V_{G S}}\right)^{2}
$$


a)

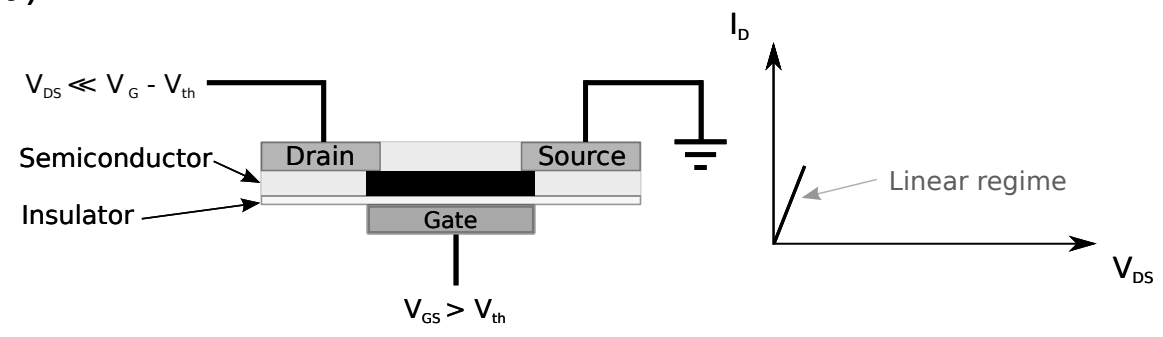

b)

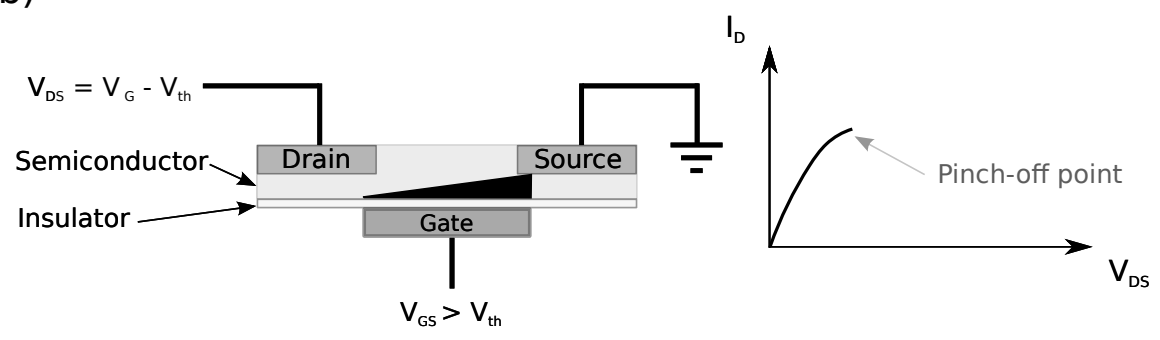

c)

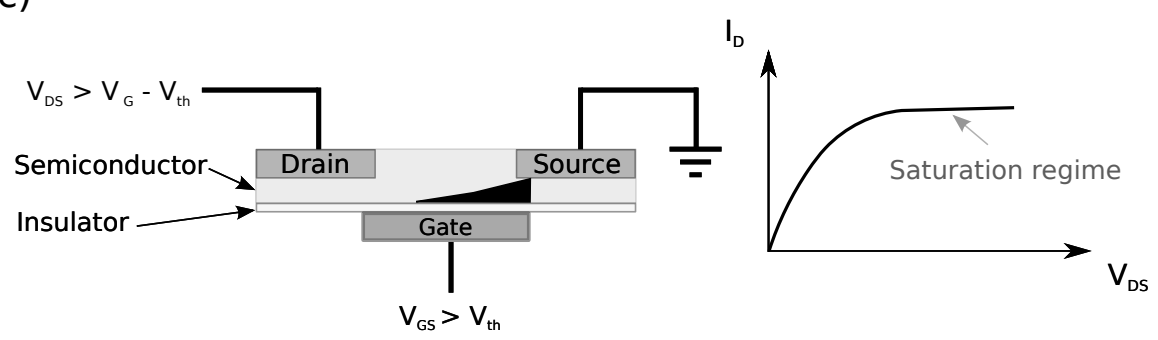

Figure 3.14: Charge distribution along the channel of a FET and corresponding current-voltage characteristics of a FET in linear regime (a), pinch-off (b) and saturation regime (c). 
Transconductance is an important FET performance metric and is defined as the ratio of the output's change in current to the input's change in potential as described by equation 3.8 .

$$
g_{m}=\frac{\partial I_{D}}{\partial V_{G S}}
$$

The transconductance in the linear regime is given by equation 3.9.

$$
g_{m}=\frac{W \mu C_{i}}{L} V_{D S}
$$

Figure 3.15 shows the current-voltage characteristics of a typical FET. The output characteristics — figure 3.15(a) — shows the drain current versus drain voltage. Each line represents a constant level of the gate voltage. The different regimes previously described in figure 3.14 are apparent.

Figure 3.15(b) represents the transfer characteristics which plots the drain current versus gate voltage as the drain voltage is held constant. The y-axis of the transfer curve is usually plotted on a logarithmic scale as on/off ratios span several orders of magnitude. The threshold voltage can be approximated by extrapolating the square root of the drain current until it crosses the x-axis. From the transfer characteristics curve, it is possible to approximate the charge mobility using equation 3.5 and 3.7 .

\subsubsection{Metal-oxide-semiconductor field-effect transistor}

The metal-oxide-semiconductor field-effect transistor (MOSFET) is, by a large margin, the most commonly encountered transistor. In fact, the seemingly inexorable development of MOSFET technology is what has sustained Moore's law over so many decades. A typical MOSFET consists of a lightly doped semiconductor (e.g. silicon) substrate that connects two highly doped regions around both the source and drain electrode. The metal in MOSFET historically refers to the material which made up the gate, source and drain electrodes, but has since been supplanted by polysilicon. The gate electrode is insulated from the channel by high-k dielectric materials (e.g. hafnium dioxide).

\subsubsection{Thin-film transistor}

A thin film transistor (TFT) has a very thin semiconducting layer, as opposed to the thicker layer in MOSFETs. TFTs are typically 
a)

b)
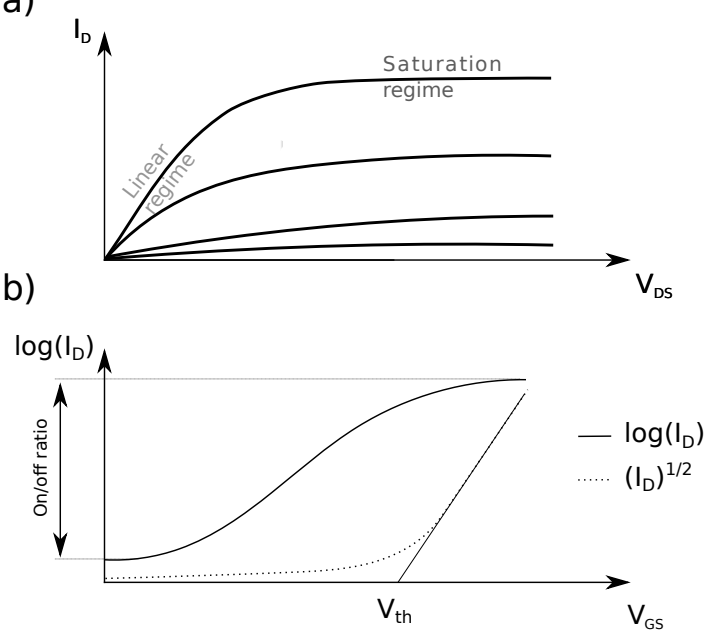

Figure 3.15: FET output (a) and transfer characteristics (b).

made on highly transmissive substates (e.g. glass), as TFTs are often used to address the pixels in flat-panel displays. If the thin layer is made from an organic semiconductor, the device is referred to as an organic thin-film transistor or, more commonly, an organic field-effect transistor.[64]

\subsubsection{Electrical double-layer organic field effect tran- sistor}

An electrical double-layer organic field transistor (EDLOFET) is a thin-film organic transistor where the insulating layer is made from a material which, when polarized, creates an electrical double layer.[55, 30] As expounded in section 3.1.2, electrical double layers are characterized by their very large capacitance per area which may reach into the hundreds of $\mu \mathrm{F} \mathrm{cm}^{-2}$. [53] Indeed, virtually all the potential drop occurs at the thin layer (on the order of the ångström) of polarized ions — i.e. Helmholtz layer. This is by opposition to the linear potential drop across a conventional dielectric layer such as the one described in section 3.1.1.1. EDLOFETs exploit this enormous capacitance by substituting the insulating layer for an EDLC.

Unlike the polarization response time of solid electrolytes, the specific capacitance of the latter is constant over a wide range of 
frequencies. [65] The capacitance in a polyelectrolyte EDLC at low frequencies is expected to be practically independent of the thickness of the electrolyte, making EDLOFETS a technology that is well-suited for printed electronics. Indeed, printing techniques such as roll-to-roll or inkjet printing suffer from subpar reproducibility and a large variation in thickness. Moreover, this large capacitance is capable of inducing a massive amount of charge carriers within the semiconducting layer ${ }^{6}$, at voltages which are relatively low $(<5 \mathrm{~V})$.

\subsubsection{Organic electrochemical transistor}

An organic electrochemical transistor (OECT) is essentially a chemiresistor, where the conductance of a chemical - organic - layer is modulated by an electrical signal.[66] The first OECT was demonstrated in 1984 and used an electropolymerized thin layer of polypyrrole.[67] Compared to an FET which relies on an electric field to function, the basis of operation of OECT is the potentialdriven electrochemical reaction.

Nowadays, OECTs are primarily made out of PEDOT:PSS. The ubiquity of the latter polymer in OECTs is primarily due to its commercial availability (in aqueous dispersion). Figure 3.16 depicts the basic mode of operation of a PEDOT:PSS OECT. Under normal circumstances, in the absence of a gate voltage (a), the PEDOT:PSS channel (i.e. between source and drain) is conducting. When applying a positive voltage to the gate, the cations in the electrolyte start to undope the PEDOT:PSS in the channel. As these cations compensate the PSS polyanion, the number of bipolarons decreases which makes the channel material less electrically conducting — see figure 3.16(b). The doping level decreases further given enough time or if a larger gate voltage is applied (c). At a certain point, the resistance of the channel becomes so large that, for all intents and purposes, it is said to be non conducting. The PEDOT:PSS gate (counter)electrode is further oxidized in a reversible fashion. However, because PEDOT:PSS is $70 \%$ oxidized in its pristine form - and given both the channel and the gate are made from PEDOT:PSS - the size of the gate must be larger than the channel such that the same amount of charges may be stored in the channel and in the gate. If the size of the gate is too small, an irreversible degradation mechanism kicks in and the PEDOT

\footnotetext{
${ }^{6}$ up to $10^{11} \mathrm{~m}^{-2}$
} 
in the channel becomes overoxidized. Because of the latter effect, special care should be taken when designing OECTs.[68] 
a)

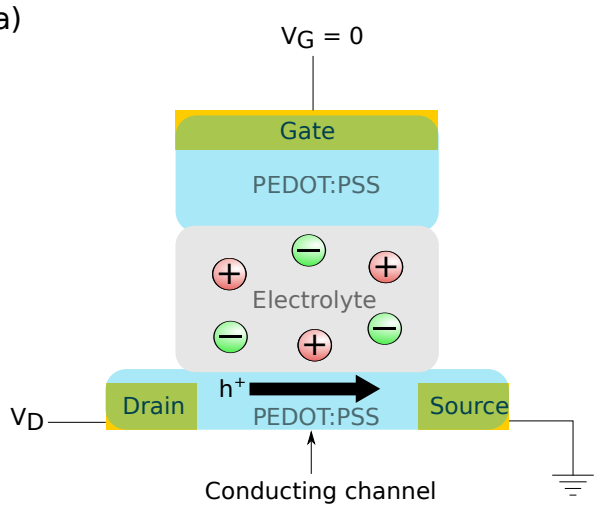

b)

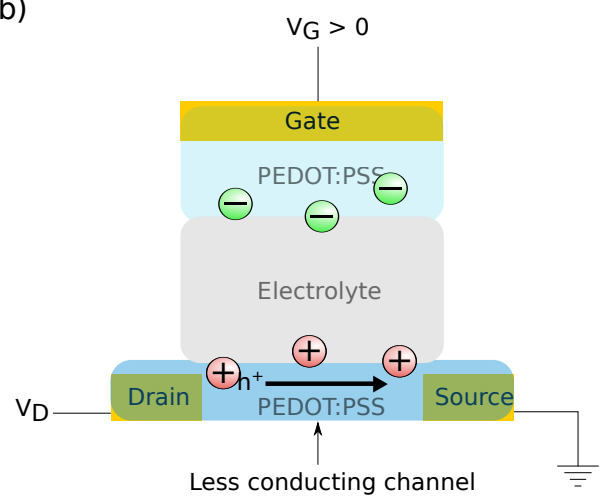

c)

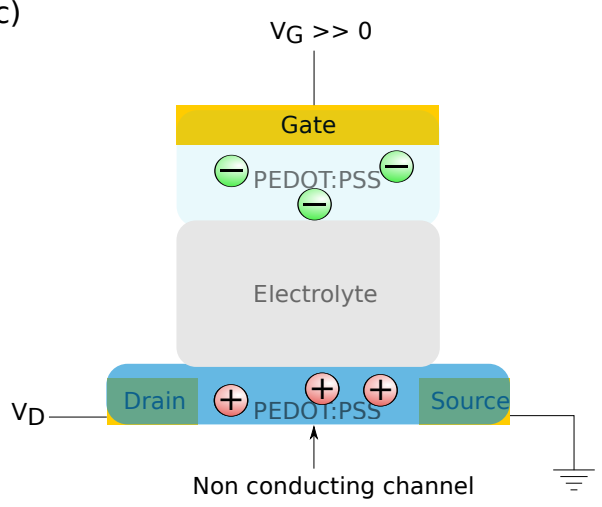

Figure 3.16: Mode of operation of a PEDOT:PSS OECT. The channel is conducting at zero gate voltage (a). Onset of channel pinch-off (b) and non-conducting channel (c). 


\section{Chapter 4}

\section{Methods}

The materials and devices developed in this thesis necessitated the use of several manufacturing and measurement techniques that this chapter attempts to describe.

\subsection{Manufacturing techniques}

\subsubsection{Spin-coating}

One of the most common methods to deposit uniform thin-films onto flat substrates is spin-coating.[69]

The machine used for this purpose consists of a spinning disk unto which the substrate is attached - typically through a vacuum system. Figure 4.1(a) depicts a spin-coating setup. The rotational speed of the disk starts in a short low-speed mode (100-1000 rpm), and moves on to 1000 to 5000 rotations per minute in the second stage of the spin-coating. The material to be deposited is dissolved in a volatile solvent and deposited onto the substrate with a pipette (see figure 4.1(b)). As the disk starts spinning, the centrifugal force exerted on the deposited solution starts to thin out the latter (see figure 4.1(c)).

After all the solvent has evaporated, a thin to ultra-thin layer of the material remains on the substrate. The thickness of the deposited material may be tuned by changing the concentration of the solution and by varying the rotational speed. Despite its extreme simplicity, the spin-coating method offers excellent reproducibility as long as the speed and air extraction are controlled for. 
a)

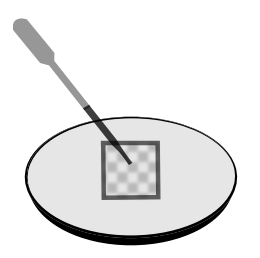

c)

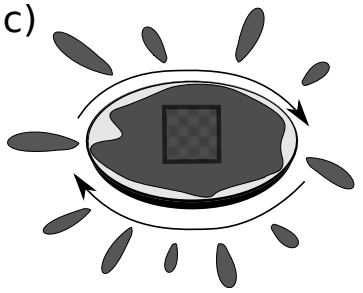

b)

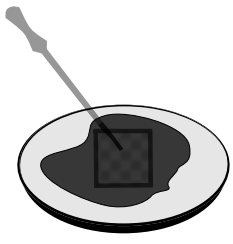

d)

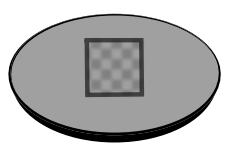

Figure 4.1: Thin-film deposition by spin-coating. The surface to be coated is affixed onto the spin-coating machine (a), the casting solution is deposited (b), the machine starts to rotate (c), and the homogeneous thin-film is obtained $(\mathrm{d})$.

However, spin-coating is a fairly wasteful method as only a fraction of the material used makes its way onto the substrate. Most of the solution gets discarded early on during the process. Moreover, the technique works best with homogeneous solutions. Minor roughness of the substrate negatively impacts the uniformity of the film thickness.

Spin-coating was used abundantly to make the devices presented in this thesis. It was used to produce homogeneous thinfilms of polymers as well as deposit photoresists (see section 4.1.2).

\subsubsection{Photolithograhy}

Photolithography is a method used to pattern a thin-film or a substrate. This method is fundamental to the fabrication of integrated circuits and microelectromechanical systems. [70] In brief, photolithography uses light to transfer a mask's pattern onto a photosensitive material called a photoresist. The processing takes place in different stages, a few of which are depicted in figure 4.2 (for a so-called positive photoresist). First, the substrate is thoroughly cleaned to remove impurities.[71] It is subsequently primed to improve adhesion to the surface. A photoresist is then spin- 
coated on it, followed by a soft-bake to make sure all the solvents have evaporated. The primary step is exposure of the substrate to light through a photomask. When exposed to UV light, a chemical reaction takes place in the positive [negative] photoresist which renders the exposed sections soluble [insoluble]. It is possible to create sub-micrometer patterns using photolithography. ${ }^{1}$

Once the exposure has been completed, a post-exposure bake reduces standing wave effects and increases the crosslinking speed of the (negative) resist.[71] Thereafter, the development step removes the unwanted resist (typically by immersion in a developer). To make sure the development process is stopped after taking the substrate out of the bath, the remaining developer is rinsed off with water. A hard-bake is then needed to remove residual solvents. The regions left unprotected by the photoresist are then etched off the substrate before the resist is thoroughly removed in the striping step.[71]

\subsubsection{Solution casting}

Solution casting (also called solvent casting) is the process whereby a solid block of material is obtained by casting a solution into a container and evaporating the solvent(s) in a controlled fashion. The container acts as a mold and is preferably made out of a non-stick surface to allow the extraction of the final product. The thickness of the desired block may be tuned by changing the concentration of the starting solution. The morphology of the obtained solid is influenced to a large degree by the drying speed. In turn, the latter is effected by the temperature, pressure and the convective heat transport.

Solution casting is useful for producing small batches of polymer composites because it requires little to no machinery.

\subsection{Electrochemistry}

Electrochemistry is the scientific discipline that studies the changes of matter effected by the flow of charge carriers (electrons, ions, etc.). If an electrical current is externally supplied to electrodes which are in contact with an electrolyte, the transfer of electrons to/from the electrodes results in reduction/oxidation reactions.

\footnotetext{
${ }^{1}$ Photolithography is limited in resolution to the wavelength of the light used. By replacing the latter with a beam of electrons, it is possible to achieve patterns with a $2 \mathrm{~nm}$ feature size.[72]
} 


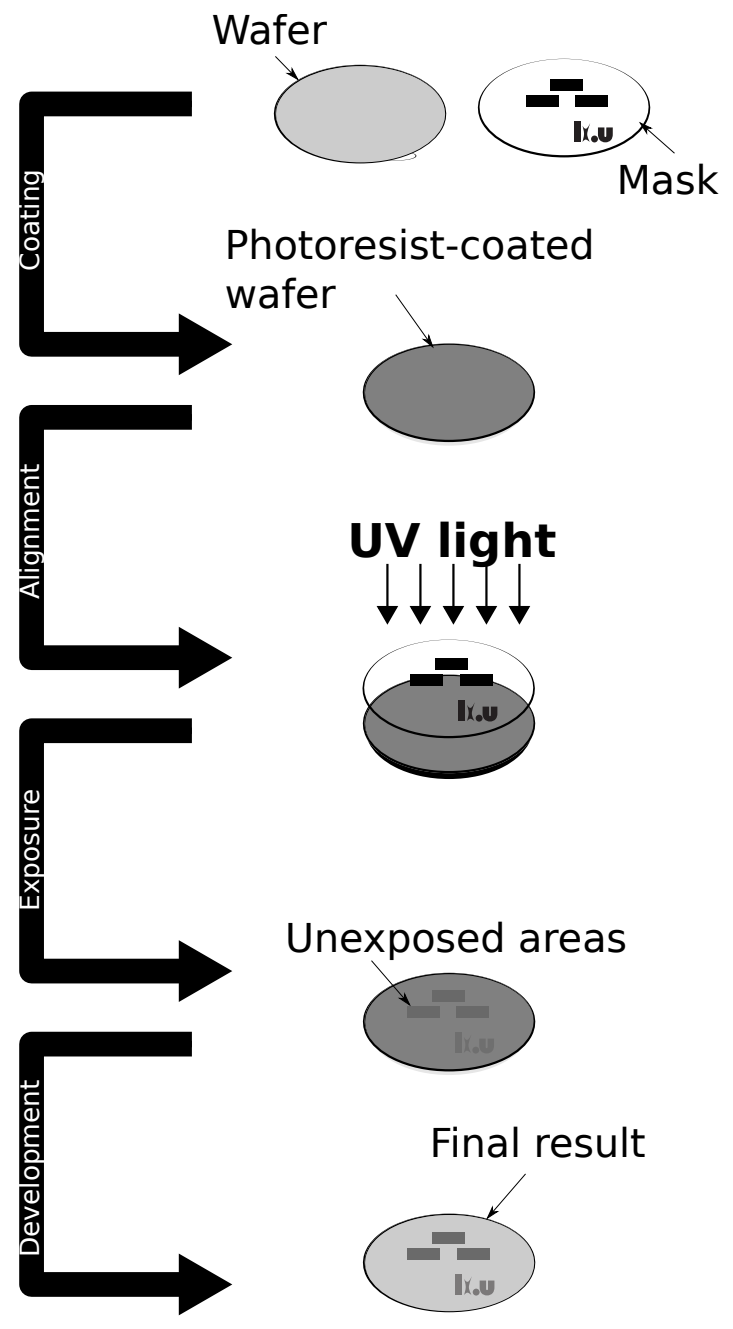

Figure 4.2: Photolithography processing steps for a positive photoresist. 


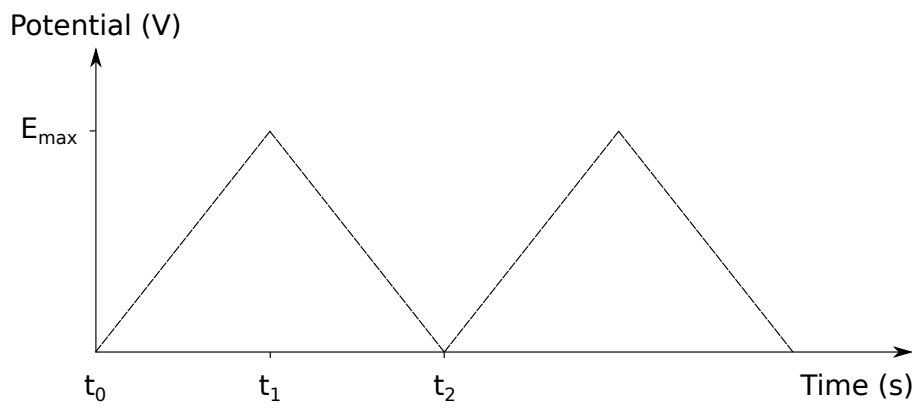

Figure 4.3: Cyclic voltammetry potential waveform.

\subsubsection{Cyclic voltammetry}

Cyclic voltammetry (CV) is one of the most widely used electrochemical techniques as it allows the qualitative study of an analyte's electrochemical properties. CV, as the name of the technique implies, cycles the potential applied across the electrodes while measuring the current. The method is useful for obtaining preliminary information about an electrochemical process.

In $\mathrm{CV}$, the potential across the electrodes is ramped linearly up to a finite level $\left(\mathrm{E}_{\max }\right)$. Once $\mathrm{E}_{\max }$ is reached, the potential is ramped down in a similar fashion. The scan-rate (expressed in $\mathrm{V} / \mathrm{s}$ ) determines the speed at which the potential is increased/decreased. Figure 4.3 shows two cycles of a typical CV scan. The first derivative (depicted as the slope of the tangent line in the graph) gives the scan-rate. Points $t_{0}, t_{1}$ and $t_{2}$ represent the start, reversal and end of the cycle. The $t_{0}-t_{1}$ region is referred to as the forward scan while $t_{1}-t_{2}$ is the reverse scan.

$\mathrm{CV}$ is typically performed using a three electrode-setup immersed in an electrolyte. It uses a potentiostat to maintain a constant change in potential difference between the working electrode and the reference electrode. CV results are typically presented in the form of an I-V characteristics curve. A CV that does not significantly deviate over time is an indication that the redox activity is reversible.

Figure 4.4 shows the typical shape of $\mathrm{CVs}$ for two electronic components - namely, a resistor (a) and a capacitor (b). The boxlike shape $\mathrm{CV}$ of a capacitor is of particular interest to this work. The current-voltage relationship in a resistor is governed by Ohm's law, which leads to the linear plot in figure 4.4(a). In a capacitor, 
a)

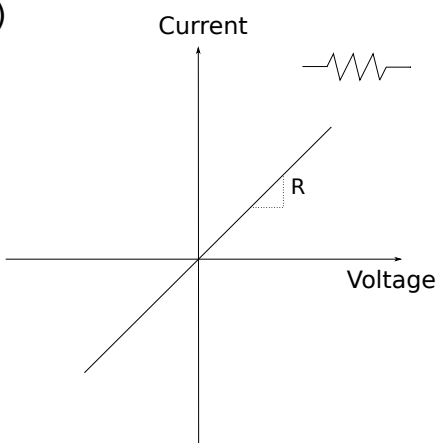

b)

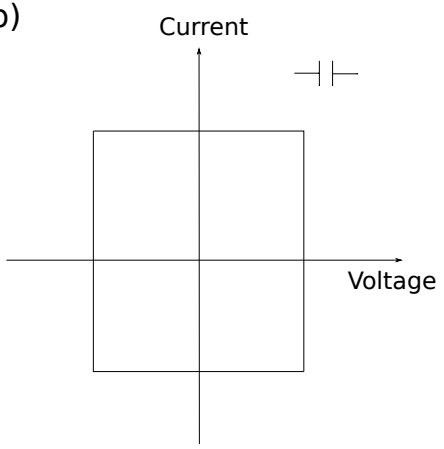

Figure 4.4: Cyclic voltammogram for a resistor (a) and a capacitor (b).

and assuming little to no faradaic processes, the current is related to the voltage by the following equation: $i(t)=C \frac{d V}{d t}$. Therefore, if the voltage is increased at a steady rate, the current will be constant and represented in the $\mathrm{CV}$ plot by a straight horizontal line. However, when the current instantaneously changes sign (as will be the case around point $t_{1}$ ) with little change in voltage, it will result in the vertical lines shown in figure 4.4(b).

The CV of systems which involve faradaic processes exhibit current peaks from which information about the nature of the reactant can be gleaned. It is important to select the scan-rate carefully as slower processes may be obscured in a $\mathrm{CV}$ performed with a fast scan-rate.

\subsubsection{Impedance spectroscopy}

Impedance spectroscopy $^{2}$ (IS) is a powerful experimental method to study the electrochemical properties of a material and the processes that take place between said material and an electrode. IS consists of measuring impedance over a wide range of frequencies at different potentials (which may include a DC component). A typical setup for performing IS is composed of two metallic electrodes sandwiching the material to be characterized. The material to be studied may be a solid or a liquid. An alternating voltage is then applied across the electrodes and the impedance is measured. This process is repeated for several frequencies and the associated

\footnotetext{
${ }^{2}$ Also called dielectric spectroscopy or electrochemical impedance spectroscopy
} 


\begin{tabular}{|l||c|c|c|}
\hline Element & I-V relationship & Impedance $(\Omega)$ & Phase angle $\left(^{\circ}\right)$ \\
\hline Resistor & $I=\frac{V}{R}$ & $Z=R$ & 0 \\
\hline Capacitor & $I=C \frac{d V}{d t}$ & $Z=\frac{1}{j \omega C}$ & -90 \\
\hline Inductor & $V=L \frac{d I}{d t}$ & $Z=j \omega C$ & 90 \\
\hline
\end{tabular}

Table 4.1: Current-voltage relationship equations, impedance expressions and phase angles for the basic circuit elements.

impedance parameters are recorded. Electrochemical cells which deviate from purely resistive behavior exhibit a phase shift between the voltage and current known as the phase angle.

Assuming a sinusoidal waveform, the applied AC potential is of the form: $V=V_{0} \sin (\omega t)$ where $\omega$ is the radial frequency $\omega=2 \pi f$. The output is shifted by a phase angle $\phi$ and, for linear systems, the current becomes: $I=I_{0} \sin (\omega t+\phi)$.

The impedance is therefore expressed in terms of a phase shift $\omega$ and a magnitude component $Z_{0}$ :

$$
Z=\frac{V_{0}}{I_{0}}=Z_{0} \frac{\sin \omega t}{\sin (\omega t+\phi)}
$$

It is also possible to express the impedance as a complex function:

$$
Z=Z_{0} e^{j \omega}
$$

The impedance data may be visualized using either the Nyquist or Bode plot. The Nyquist plot represents the imaginary (capacitive and/or inductive) components of the cell versus the real (resistive) part. The Bode plot is a direct representation of the magnitude $\left(\mathrm{Z}_{0}\right)$ and phase angle $(\omega)$ versus frequency. The frequency and magnitude of the impedance are most often plotted on a logarithmic scale.

Table 4.1 summarizes a few important IS-related parameters for the ideal resistor, capacitor and inductor.

Most electrochemical cells can be described using only these basic circuit elements. If those elements fail to fully describe a system, one may resort to the Warburg diffusion element which has a phase angle of $-45^{\circ}$ and the following magnitude of impedance: $Z_{w}=\frac{A_{w}}{\sqrt{(j \omega)}}$. In this case, $\mathrm{A}_{\mathrm{w}}$ is the Warburg constant which is used to model the diffusion of ions. 


\section{Chapter 5}

\section{Conclusions}

For well over a decade, printed electronics has been hailed as the technology which will enable ubiquitous and low-cost electronic devices. $[4,73]$ The organic and printed electronics market has been estimated to exceed $\$ 300$ billion over the next 15 years. Yet, few (if any) printed electronics commercial ventures managed to compete against the more traditional processes to produce electronics. [74] These failures have been ascribed to the fabrication processes' immaturity or lack of a killer app.

This is a dubious explanation considering the astonishing quantities of good food that are discarded because we lack a way to efficiently assess spoilage, and that printed sensing circuitry that tracks every individual package as it makes its way down the supply chain would greatly reduce food waste.[75, 76, 77] Adapting printers to accommodate conducting inks should be considered a trivial task considering that printing technology goes back to the dawn of the industrial revolution. A stronger case for the slow adoption can be made if one considers the absence of versatile ink formulations. One of the field's priorities was stated as "to find one mechanism or switch phenomena in a material system that can modulate charge transport, be used in displays, and be used for power storage or conversion".[78]

The present work attempted to reconcile organic electronics with very thick-film $(>100 \mu \mathrm{m})$ technologies. First, thin-films $(<200 \mathrm{~nm})$ of organic materials were combined with an electric double layer capacitor to decrease the operating voltage of organic field effect transistors [Paper I and II]. Depositing the insulating layer is also simplified when using polyelectrolyte EDLCs, 
because the latter's capacitance is independent of thickness. [65] Later on, the use of polyelectrolyte layers of opposite polarities (bipolar membrane) was investigated to produce (ionic) currentrectifying diodes. These bipolar membranes were integrated inside electrochromic displays [Paper III]. This integration is particularly interesting because the production process of bipolar membranes is fully-compatible with existing (customized) printing machinery and manufacturing techniques. We demonstrated that by adding a single layer of oppositely-charged polyelectrolyte to the configuration of conventional printed electrochromic displays, we were able to increase the device's bistability and obviate the need for an expensive addressing backplane. Moreover, all the active materials (i.e. besides the substrate) used in this display were processable from aqueous suspensions/solutions.

Thereafter, the possibility to forgo the substrate in a variety of devices was demonstrated. It is possible to produce a selfstanding electrochromic thick-film $(>100 \mu \mathrm{m})$ by compositing the same water-processable material with nanofibrillated cellulose (plus a whitening pigment and high-boiling point solvents) [Paper IV]. This was used in conjunction with a polyelectrolyte to fabricate a substrate-free organic electrochromic device. The cellulose nanofibrils were found to be coated with the conducting polymer, while acting as the scaffold which gave it structural integrity. The voids created at the intersection of these nanofibrils appear to be filled with high-boiling point solvents, which confer the composite a good ionic conductivity.

In addition, the viability of these (semi)conducting polymer nanopaper composites in a variety of applications was investigated [Paper V]. This material exhibited an excellent combined electronicionic conductivity. Moreover, the conductivities in this easy-toprocess composite remained constant within a wide range of thicknesses. Initially, it was used to produce electrochemical transistors with a giant transconductance $(>1 \mathrm{~S})$ which, at the time of writing, is a two orders of magnitude improvement on the state-ofthe-art.[79] This feat was possible by scaling up the thickness of the transistor channel's geometry. Subsequently, these (semi)conducting nanopaper composites were used as electrodes in a supercapacitor and were able to routinely produce devices whose capacitance exceeds $1 \mathrm{~F}$. All the while, this composite may carry a current density above $1 \mathrm{~A} \mathrm{~mm}^{-2}$. Given that copper is the most hazardous of all metals to human health and ecosystem damage (in absolute terms), conducting polymer composites may become an attractive 
alternative in several applications.[80]

To rise above the level of mere gimmickry, the field of printed and organic electronics needs to focus on materials which are scalable and easily processable. With the development of ever-more flexible glass (e.g. the Corning Willow series), organic and printed electronics will have to move beyond mere device flexibility. Rather, the focus should be on flexible and scalable production methods. Exploiting the unique properties of nanofibrillated cellulose (excellent mechanical properties, high aspect ratio, etc.) to bind and even orient conducting polymers opens the door for a vast array of applications. 


\section{Bibliography}

[1] B. J. Pine. Mass customization: the new frontier in business competition. Harvard Business Press, 1999.

[2] C. K. Chiang, C. R. Fincher, Y. W. Park, A. J. Heeger, H. Shirakawa, E. J. Louis, S. C. Gau, and A. G. MacDiarmid. Electrical conductivity in doped polyacetylene. Physical Review Letters, 39(17):1098-1101, 1977.

[3] A. J. Heeger. Semiconducting and metallic polymers: The fourth generation of polymeric materials (nobel lecture). Angewandte Chemie - International Edition, 40(14):25912611, 2001.

[4] S. R. Forrest. The path to ubiquitous and low-cost organic electronic appliances on plastic. Nature, 428(6986):911-918, 2004.

[5] M. Irimia-Vladu. Green electronics: biodegradable and biocompatible materials and devices for sustainable future. Chem. Soc. Rev., 43:588-610, 2014.

[6] T. G. Gutowski, M. S. Branham, J. B. Dahmus, A. J. Jones, A. Thiriez, and D. P. Sekulic. Thermodynamic analysis of resources used in manufacturing processes. Environmental Science and Technology, 43(5):1584-1590, 2009.

[7] P. V. Wright. Polymer electrolytes - the early days. Electrochimica Acta, 43(10-11):1137-1143, 1998.

[8] M. Armand. Polymer solid electrolytes - an overview. Solid State Ionics, 9-10(PART 2):745-754, 1983.

[9] M. Armand. Polymers with ionic conductivity. Advanced Materials, 2(6-7):278-286, 1990. 
[10] D. T. Hallinan Jr. and N. P. Balsara. Polymer electrolytes, volume 43 of Annual Review of Materials Research. 2013.

[11] V. Di Noto, S. Lavina, G. A. Giffin, E. Negro, and B. Scrosati. Polymer electrolytes: Present, past and future. Electrochimica Acta, 57(1):4-13, 2011.

[12] G. T. Sten. Bag with handle of weldable plastic material, 1965. US Patent 3,180,557.

[13] P. P. W. Atkins and J. De Paula. Atkins' Physical Chemistry. Oxford University Press, Incorporated, 2002.

[14] M. Fahlman and W. R. Salaneck. Surfaces and interfaces in polymer-based electronics. Surface Science, 500(1-3):904-922, February 2002.

[15] Handbook of Advanced Electronic and Photonic Materials and Devices. Academic Press.

[16] I. Kang, H. Yun, D. S. Chung, S. Kwon, and Y. Kim. Record high hole mobility in polymer semiconductors via sidechain engineering. Journal of the American Chemical Society, 135(40):14896-14899, 2013.

[17] M. Vissenberg and M. Matters. Theory of the field-effect mobility in amorphous organic transistors. Physical Review $B$ - Condensed Matter and Materials Physics, 57(20):1296412967, 1998.

[18] R. J. Mortimer. Electrochromic materials, volume 41 of Annual Review of Materials Research. 2011.

[19] S. K. Deb. Optical and photoelectric properties and colour cetres in thin films of tungsten oxide. Phil Mag, 17(4):801822, 1973.

[20] D. T. Gillaspie, R. C. Tenent, and A. C. Dillon. Metal-oxide films for electrochromic applications: present technology and future directions. J. Mater. Chem., 20:9585-9592, 2010.

[21] P. Monk, R. Mortimer, and D. Rosseinsky. Electrochromism and Electrochromic Devices. Cambridge University Press, 2007.

[22] C. M. Lampert. Chromogenic smart materials. Materials Today, 7(3):28-35, 2004. 
[23] C. Hamann, A. Hamnett, and W. Vielstich. Electrochemistry. Wiley-VCH, 2007.

[24] T. Torimoto, T. Tsuda, K. Okazaki, and S. Kuwabata. New frontiers in materials science opened by ionic liquids. Advanced Materials, 22(11):1196-1221, 2010.

[25] S. H. Kim, K. Hong, W. Xie, K. H. Lee, S. Zhang, T. P. Lodge, and C. D. Frisbie. Electrolyte-gated transistors for organic and printed electronics. Advanced Materials, 25(13), 2013.

[26] A. Manuel Stephan. Review on gel polymer electrolytes for lithium batteries. European Polymer Journal, 42(1):21 - 42, 2006 .

[27] A. Kaltbeitzel, S. Schauff, H. Steininger, B. Bingöl, G. Brunklaus, W. H. Meyer, and H. W. Spiess. Water sorption of poly(vinylphosphonic acid) and its influence on proton conductivity. Solid State Ionics, 178(7-10):469 - 474, 2007. 13th Solid State Proton Conductors Conference.

[28] J. Yuan, D. Mecerreyes, and M. Antonietti. Poly(ionic liquid)s: An update. Progress in Polymer Science, 38(7):10091036, 2013.

[29] K. Campbell, M. Yanez Arteta, A. Angus-Smyth, T. Nylander, and I. Varga. Effects of bulk colloidal stability on adsorption layers of poly(diallyldimethylammonium chloride)/sodium dodecyl sulfate at the air-water interface studied by neutron reflectometry. The Journal of Physical Chemistry B, 115(51):15202-15213, 2011.

[30] A. Malti, M. Berggren, and X. Crispin. Low-voltage ambipolar polyelectrolyte-gated organic thin film transistors. Applied Physics Letters, 100(18), 2012.

[31] D. S. Peterson. Ion exchange membranes. In Dongqing Li, editor, Encyclopedia of Microfluidics and Nanofluidics, pages 1-5. Springer US, 2014.

[32] R. K. Nagarale, G. S. Gohil, and V. K. Shahi. Recent developments on ion-exchange membranes and electro-membrane processes. Advances in Colloid and Interface Science, 119(2-3):97 - 130, 2006. 
[33] K. Tybrandt, E. O. Gabrielsson, and M. Berggren. Toward complementary ionic circuits: The npn ion bipolar junction transistor. Journal of the American Chemical Society, 133(26):10141-10145, 2011.

[34] A. Malti, E. O. Gabrielsson, X. Crispin, and M. Berggren. An electrochromic bipolar membrane diode. Advanced Materials, 27(26):3909-3914, 2015.

[35] Y. Habibi, L. A. Lucia, and O. J. Rojas. Cellulose nanocrystals: chemistry, self-assembly, and applications. Chemical reviews, 110(6):3479-3500, 2010.

[36] D. Tobjörk and R. Österbacka. Paper electronics. Advanced Materials, 23(17):1935-1961, 2011.

[37] Deutsche Technikmuseum. Papiertechnik (permament exhibition). Berlin, 2015.

[38] D. Klemm, B. Heublein, H. Fink, and A. Bohn. Cellulose: Fascinating biopolymer and sustainable raw material. Angewandte Chemie International Edition, 44(22):3358-3393, 2005.

[39] R. Moon, A. Martini, J. Nairn, J. Simonsen, and J. Youngblood. Cellulose nanomaterials review: structure, properties and nanocomposites. Chem. Soc. Rev., 40:3941-3994, 2011.

[40] Stone age, iron age, polymer age. Journal of Chemical Education, 63(9):743, 1986.

[41] R. Bollström, A. Määttänen, D. Tobjörk, P. Ihalainen, N. Kaihovirta, R. Österbacka, J. Peltonen, and M. Toivakka. A multilayer coated fiber-based substrate suitable for printed functionality. Organic Electronics, 10(5):1020 - 1023, 2009.

[42] H. Sixta. Handbook of Pulp. Wiley-VCH Verlag GmbH, 2008.

[43] S. Kalia, S. Boufi, A. Celli, and S. Kango. Nanofibrillated cellulose: surface modification and potential applications. Colloid and Polymer Science, 292(1):5-31, 2014.

[44] M. Henriksson, L. A. Berglund, P. Isaksson, T. Lindström, and T. Nishino. Cellulose nanopaper structures of high toughness. Biomacromolecules, 9(6):1579-1585, 2008. 
[45] V. Favier, H. Chanzy, and J. Y. Cavaille. Polymer nanocomposites reinforced by cellulose whiskers. Macromolecules, 28(18):6365-6367, 1995.

[46] R. Olsson, A. Samir, G G. Salazar-Alvarez, L. Belova, V. Ström, L. A. Berglund, O. Ikkala, J. Nogues, and U. W. Gedde. Making flexible magnetic aerogels and stiff magnetic nanopaper using cellulose nanofibrils as templates. Nature nanotechnology, 5(8):584-588, 2010.

[47] N. Lin and A. Dufresne. Nanocellulose in biomedicine: Current status and future prospect. European Polymer Journal, 59:302-325, 2014.

[48] C. Eyholzer, A. Borges de Couraça, F. Duc, P. E. Bourban, P. Tingaut, T. Zimmermann, J. A. E. Månson, and K. Oksman. Biocomposite hydrogels with carboxymethylated, nanofibrillated cellulose powder for replacement of the nucleus pulposus. Biomacromolecules, 12(5):1419-1427, 2011.

[49] H. Valo, M. Kovalainen, P. Laaksonen, M. Häkkinen, S. Auriola, L. Peltonen, M. Linder, K. Järvinen, J. Hirvonen, and T. Laaksonen. Immobilization of protein-coated drug nanoparticles in nanofibrillar cellulose matrices - enhanced stability and release. Journal of Controlled Release, 156(3):390 - 397, 2011.

[50] P. Simon and Y. Gogotsi. Materials for electrochemical capacitors. Nature materials, 7(11):845-854, 2008.

[51] B. E. Conway. Electrochemical supercapacitors: scientific fundamentals and technological applications. Springer Science \& Business Media, 2013.

[52] O. Stern. Zur theorie der elektrolytischen doppelschicht. Zeitschrift für Elektrochemie und angewandte physikalische Chemie, 30(21-22):508-516, 1924.

[53] S Mitra, A.K Shukla, and S Sampath. Electrochemical capacitors with plasticized gel-polymer electrolytes. Journal of Power Sources, 101(2):213 - 218, 2001.

[54] A. J. Bard and L. R. Faulkner. Electrochemical methods: fundamentals and applications, volume 2. Wiley New York, 1980. 
[55] L. Herlogsson, X. Crispin, N. D. Robinson, M. Sandberg, O.-J. Hagel, G. Gustafsson, and M. Berggren. Low-voltage polymer field-effect transistors gated via a proton conductor. Advanced Materials, 19(1):97-101, 2007.

[56] J. H. Cho, J. Lee, Y. Xia, B. Kim, Y. He, M. J. Renn, T. P. Lodge, and C. D. Frisbie. Printable ion-gel gate dielectrics for low-voltage polymer thin-film transistors on plastic. Nature Materials, 7(11):900-906, 2008.

[57] S. Abbrent and S. Greenbaum. Polymer Electrolytes. Woodhead Publishing Series in Electronic and Optical Materials. Woodhead Publishing, 2010.

[58] J. C. Carlberg and O. Inganäs. Poly (3, 4ethylenedioxythiophene) as electrode material in electrochemical capacitors. Journal of the Electrochemical Society, 144(4):L61-L64, 1997.

[59] S. Sapp, G. A. Sotzing, J. L. Reddinger, and J. R. Reynolds. Rapid switching solid state electrochromic devices based on complementary conducting polymer films. Advanced Materials, 8(10):808-811, 1996.

[60] J. Kawahara, P. Andersson Ersman, I. Engquist, and M. Berggren. Improving the color switch contrast in pedot:pssbased electrochromic displays. Organic Electronics, 13(3):469 $-474,2012$.

[61] P. Horowitz and W. Hill. The art of electronics. Cambridge Univ. Press, 1989.

[62] J. Lilienfeld. Method and apparatus for controlling electric currents, 1930. US Patent 1,745,175.

[63] H. Heil. Improvements in or relating to electrical amplifiers and other control arrangements and devices, 1935. UK Patent 439,457 .

[64] Y Yangand A. J. Heeger. A new architecture for polymer transistors. Nature, 372(6504):344-346, 1994.

[65] K. H. Lee, S. Zhang, T. P. Lodge, and C. D. Frisbie. Electrical impedance of spin-coatable ion gel films. The Journal of Physical Chemistry B, 115(13):3315-3321, 2011. 
[66] H. Wohltjen. Chemical microsensors and microinstrumentation. Analytical Chemistry, 56(1):87A-103A, 1984.

[67] H. S. White, G. P. Kittlesen, and M. S. Wrighton. Chemical derivatization of an array of three gold microelectrodes with polypyrrole: fabrication of a molecule-based transistor. Journal of the American Chemical Society, 106(18):5375-5377, 1984 .

[68] P. Tehrani, A. Kanciurzewska, X. Crispin, N. D. Robinson, M. Fahlman, and M. Berggren. The effect of ph on the electrochemical over-oxidation in pedot:pss films. Solid State Ionics, 177(39-40):3521 - 3527, 2007.

[69] C. J. Lawrence. The mechanics of spin coating of polymer films. Physics of Fluids, 31(10):2786-2795, 1988.

[70] J. B. Waldner. Nanocomputers and Swarm Intelligence. 2010.

[71] C. Mack. Fundamental Principles of Optical Lithography: The Science of Microfabrication. 2007.

[72] V. R. Manfrinato, L. Zhang, D. Su, H. Duan, R. G. Hobbs, E. A. Stach, and K. K. Berggren. Resolution limits of electronbeam lithography toward the atomic scale. Nano Letters, 13(4):1555-1558, 2013.

[73] D. Tobjörk and R. Österbacka. Paper electronics. Advanced Materials, 23(17):1935-1961, 2011.

[74] N. C. Karmakar. Chipless and conventional radio frequency identification: Systems for ubiquitous tagging. 2012.

[75] J. Parfitt, M. Barthel, and S. MacNaughton. Food waste within food supply chains: Quantification and potential for change to 2050. Philosophical Transactions of the Royal Society B: Biological Sciences, 365(1554):3065-3081, 2010.

[76] H. C. J. Godfray, J. R. Beddington, I. R. Crute, L. Haddad, D. Lawrence, J. F. Muir, J. Pretty, S. Robinson, S. M. Thomas, and C. Toulmin. Food security: The challenge of feeding 9 billion people. Science, 327(5967):812-818, 2010.

[77] J. A. Foley, N. Ramankutty, K. A. Brauman, E. S. Cassidy, J. S. Gerber, M. Johnston, N. D. Mueller, C. O'Connell, D. K. Ray, P. C. West, C. Balzer, E. M. Bennett, S. R. Carpenter, 
J. Hill, C. Monfreda, S. Polasky, J. Rockstroem, J. Sheehan, S. Siebert, D. Tilman, and D. P. M. Zaks. Solutions for a cultivated planet. Nature, 478(7369):337-342, 2011.

[78] M. Berggren, D. Nilsson, and N. D. Robinson. Organic materials for printed electronics. Nature Materials, 6(1):3-5, 2007.

[79] D. Khodagholy, J. Rivnay, M. Sessolo, M. Gurfinkel, P. Leleux, L. H. Jimison, E. Stavrinidou, T. Herve, S. Sanaur, and O. M. Róisín. High transconductance organic electrochemical transistors. Nature communications, 4, 2013.

[80] P. Nuss and M. J. Eckelman. Life cycle assessment of metals: A scientific synthesis. PLoS ONE, 2014. 


\section{Part II}

\section{Scholarly articles}





\section{Paper I}

\section{Ultra-low Voltage Air-stable Polyelectrolyte Gated n-type Organic Thin Film Transis- tors}

A. Malti, E. O. Gabrielsson, M. Berggren, X. Crispin

Appl. Phys. Lett. 99, 063305 (2011) 



\section{Paper II}

Low-voltage Ambipolar Polyelectrolyte-gated Organic Thin Film Transistors

A. Malti, M. Berggren, X. Crispin

Appl. Phys. Lett. 100, 183302 (2012) 



\section{Paper III}

An electrochromic Bipolar Membrane Diode

A. Malti, E.O. Gabrielsson, X. Crispin, M. Berggren

Adv. Mater. 27, 3909-3914 (2015) 



\section{Paper IV}

\section{A substrate-free electrochromic device}

A. Malti, R. Brooke, X. Liu, D. Zhao, P. Andersson Ersman, M. Fahlman, M. Berggren, X. Crispin

Submitted (Aug. 2015) 



\section{Paper V}

Enabling organic power electronics with a cellulose nano-scaffold

A. Malti, J. Edberg, H. Granberg, Z. Khan, J. Andreasen, X. Liu, D. Zhao, H. Zhang, Y. Yao, J. Brill, I. Engquist, M. Fahlman, L. Wåberg, X. Crispin, M. Berrgren

Submitted (Sept. 2015) 



\section{Papers}

The articles associated with this thesis have been removed for copyright reasons. For more details about these see:

http://urn.kb.se/resolve?urn=urn:nbn:se:liu:diva-122022 